\title{
Structural GARCH: The Volatility-Leverage Connection
}

\section{Citation}

Engle, Robert F., and Emil N. Siriwardane. "Structural GARCH: The Volatility-Leverage

Connection." Harvard Business School Working Paper, No. 16-009, July 2015.

\section{Permanent link}

http://nrs.harvard.edu/urn-3:HUL.InstRepos:17527682

\section{Terms of Use}

This article was downloaded from Harvard University's DASH repository, and is made available under the terms and conditions applicable to Open Access Policy Articles, as set forth at http:// nrs.harvard.edu/urn-3:HUL.InstRepos:dash.current.terms-of-use\#OAP

\section{Share Your Story}

The Harvard community has made this article openly available.

Please share how this access benefits you. Submit a story.

\section{Accessibility}




\section{Structural GARCH: The Volatility- Leverage Connection}

Robert Engle

Emil Siriwardane

Working Paper 16-009 


\section{Structural GARCH: The Volatility- Leverage Connection}

Robert Engle

NYU Stern School of Business

Emil Siriwardane

Harvard Business School

Working Paper 16-009 not be reproduced without permission of the copyright holder. Copies of working papers are available from the author. 


\title{
Structural GARCH: The Volatility-Leverage
}

\section{Connection*}

\author{
Robert Engle $^{\dagger} \quad$ Emil Siriwardane $e^{\ddagger}$
}

July 17, 2015

\begin{abstract}
We propose a new model of volatility where financial leverage amplifies equity volatility by what we call the "leverage multiplier." The exact specification is motivated by standard structural models of credit; however, our parameterization departs from the classic Merton (1974) model and can accommodate environments where the firm's asset volatility is stochastic, asset returns can jump, and asset shocks are nonnormal. In addition, our specification nests both a standard GARCH and the Merton model, which allows for a statistical test of how leverage interacts with equity volatility. Empirically, the Structural GARCH model outperforms a standard asymmetric GARCH model for approximately 74 percent of the financial firms we analyze. We then apply the Structural GARCH model to two empirical applications: the leverage effect and systemic risk measurement. As a part of our systemic risk analysis, we define a new measure called "precautionary capital" that uses our model to quantify the advantages of regulation aimed at reducing financial firm leverage.
\end{abstract}

${ }^{*}$ We are grateful to Viral Acharya, Rui Albuquerque, Tim Bollerslev, Gene Fama, Xavier Gabaix, Paul Glasserman, Lars Hansen, Bryan Kelly, Andy Lo, and Eric Renault for valuable comments and discussions, and to seminar participants at AQR Capital Management, the Banque de France, ECB MaRS 2014, the University of Chicago (Booth), the MFM Fall 2013 Meetings, the Office of Financial Research (OFR), NYU Stern, and the WFA (2014). We are also extremely indebted to Rob Capellini for all of his help on this project. The authors thankfully acknowledge financial support from the Sloan Foundation. The views expressed in this paper are those of the authors' and do not necessarily reflect the position of the Office of Financial Research (OFR), or the U.S. Treasury Department.

${ }^{\dagger}$ Engle: NYU Stern School of Business. Address: 44 West 4th St., Suite 9-62. New York, NY 10012. E-mail: rengle@stern.nyu.edu

¥Siriwardane: NYU Stern School of Business, and the Office of Financial Research, U.S. Treasury Department. Address: 44 West 4th St., Floor 9-Room 197G. New York, NY 10012. E-mail: esiriwar@stern.nyu.edu or emil.siriwardane@treasury.gov. 


\section{Introduction}

The financial crisis revealed the damaging role of financial market leverage on the real economy. Nonetheless, it is far from clear that reducing this leverage will stabilize the real economy, let alone stabilize the financial sector. The extreme volatility of asset prices was a joint consequence of the high impact of economic news and high leverage. A critical question that remains is how much reduction in equity volatility could be expected from reductions in leverage.

To answer this question, this paper develops a model of equity volatility that reflects firm leverage. The model is motivated by the structural models of credit that follow from Merton (1974). ${ }^{1}$ However our model extends beyond the classic Merton model to accommodate jumps and stochastic volatility. In our model, asset volatility is stochastic and fat tailed. It is amplified by a "leverage multiplier" to yield equity market volatility. The parameters of the model are estimated by maximum likelihood and can be used to decompose equity volatility into the part due to asset volatility and the part due to leverage. The promise of structural credit models for volatility modeling is related to the Schaefer and Strebulaev (2008) observation that hedge ratios are well modeled by structural models of credit risk.

As with all structural models, we begin from the observation that equity is ultimately a call option on the asset value of the firm, but that the appropriate option pricing formula is dependent on the structure of the unobservable asset value. We then examine a range of economic models that differ in how asset values evolve, leading us to specify an empirical model that approximately nests the candidate option formulae. The econometric model entails parameterizing the leverage multiplier as a function of the moneyness of the option

\footnotetext{
${ }^{1} \mathrm{~A}$ non-exhaustive list of theoretical extensions of the Merton (1974) model includes Black and Cox (1976), Leland and Toft (1996), Collin-Dufresne and Goldstein (2001), and McQuade (2013).
} 
and the volatility over the life of the debt, without requiring the unobservable asset value. We call this specification a Structural GARCH model given its theoretical underpinnings.

Our empirical results show that incorporating leverage via the leverage multiplier, as in our Structural GARCH model, outperforms a simple vanilla asymmetric GARCH model of equity returns. $^{2}$ An additional advantage of our model is that it nests a vanilla GARCH, and thus provides a natural way to assess the statistical significance of leverage for equity volatility. ${ }^{3}$ For the sample of firms we examine, nearly 74 percent favor our Structural GARCH model over a plain asymmetric GARCH model. Since the Structural GARCH model delivers a daily series for asset volatility, we are also able to study the joint dynamics of asset volatility and leverage in the build up to the financial crisis. The empirical results reveal that at the onset of the financial crisis, the rise in equity volatility was primarily due to rising leverage, but later phases also include substantial rises in asset volatility.

To further demonstrate the usefulness of our econometric model, we apply the Structural GARCH model to two applications: determining the sources of asymmetric equity volatility and measuring systemic risk. Equity volatility asymmetry refers to the well-known negative correlation between equity returns and equity volatility. One popular explanation for this empirical regularity is leverage. Namely, when a firm experiences negative equity returns, its leverage mechanically rises, and thus the firm has more risk (volatility). Some examples of previous work on this topic include Black (1976), Christie (1982), French, Schwert, and Stambaugh (1987), and Bekaert and Wu (2000). The challenge faced in previous studies is that asset returns are unobservable, so teasing out the causes of volatility asymmetry requires

\footnotetext{
${ }^{2}$ There is a vast literature on ARCH/GARCH models, starting with Engle (1982) and Bollerslev (1986).

${ }^{3}$ Indeed, asymmetric GARCH models have been interpreted as capturing the interaction between leverage and equity volatility. This is famously known as the "leverage effect" of Black (1976) and Christie (1982). In contrast, our model directly incorporates volatility asymmetry at the asset level and also directly incorporates leverage into equity volatility. As we will discuss shortly, this also allows us to tease out the root of the observed leverage effect.
} 
alternative strategies. For instance, Choi and Richardson (2012) approach this problem by invoking the second Modigliani-Miller theorem. In turn, they directly compute the market value of assets at a monthly frequency by first constructing a return series for the market value of bonds (and loans). Still, determining the true market value of the bonds of a given firm is difficult in lieu of liquidity issues, especially at the daily frequency with which our model operates.

On the other hand, the Structural GARCH model provides a simpler way to estimate asset returns and volatility, while crucially allowing for the debt of each firm to be risky. Given that we obtain asymmetric GARCH parameter estimates for daily asset returns, our model provides a novel way to explore the root of the leverage effect. ${ }^{4}$ We find that, on average, firms with more leverage exhibit a bigger gap between the asymmetry of their equity return volatility and their asset return volatility. Nonetheless, our findings suggest that the overall contribution of measured leverage to the so-called "leverage effect" is somewhat weak; for our sample of firms, leverage accounts for only about 17 percent of equity volatility asymmetry. ${ }^{5}$

The second application of our Structural GARCH model involves systemic risk measurement. We extend the SRISK measure of Acharya, Pedersen, Philippon, and Richardson (2012) and Brownlees and Engle (2012) by incorporating the Structural GARCH model for firm-level equity returns. The SRISK measure captures how much capital a firm would need in the event of another financial crisis, where a financial crisis is defined as a 40 percent

\footnotetext{
${ }^{4}$ Also, in contrast to previous work, our model directly incorporates risky debt into the equity return specification. The emphasis of risky debt is important, since it introduces important nonlinear interactions between leverage and equity volatility.

${ }^{5}$ These results are consistent with Bekaert and Wu (2000) and Hasanhodzic and Lo (2013) who find that leverage does not appear to fully explain the asymmetry in equity volatility. Bekaert and Wu (2000), however, assume that debt is riskless and are therefore silent about the nonlinear interaction between equity volatility and leverage. Hasanhodzic and Lo (2013) focus on a subset of firms with no leverage, which we do not pursue in this paper. The results in Choi and Richardson (2012) are also consistent with our cross-sectional results, but they find evidence of a larger contribution of leverage to the leverage effect.
} 
decline in the aggregate stock market over a six month period. Importantly, the leverage amplification mechanism built into our model naturally embeds the types of volatility-leverage spirals observed during the crisis. It is precisely this feature that makes leverage an important consideration even in times of low volatility, since a negative sequence of equity returns increases leverage and further amplifies negative shocks to assets. Accordingly, we show that using the Structural GARCH model for systemic risk measurement shows promise in providing earlier signals of financial firm distress. Compared with models that do not incorporate leverage amplifications explicitly, the model-implied expected capital shortfall in a crisis for firms, such as Citibank and Bank of America, rises much earlier prior to the financial crisis (and remains as high or higher through the crisis). Thus, Structural GARCH serves as an important step towards developing countercyclical measures of systemic risk that may also motivate policies which prevent excess leverage from building within the financial system.

To this end, we then propose a new measure of systemic risk which we call precautionary capital. Precautionary capital is the answer to the question: how much equity do we have to add to a firm today in order to ensure some arbitrary level of confidence that the firm will not go bankrupt in a future crisis? In the Structural GARCH model, we then show that preventative measures that reduce leverage can be very powerful since holding more capital results in lower volatility, lower beta, and lower probability of failure. This is a sensible outcome that is not implied by conventional volatility models.

Section 2 introduces the Structural GARCH model and its economic underpinnings. Here, we will use basic ideas from structural models of credit to explore the relationship between leverage and equity volatility, which leads to a natural econometric specification for equity and asset returns. In Section 3, we describe the data used in our empirical work, along with some technical issues regarding estimation of the model. Section 4 describes our empirical 
results and explores some aggregate implications of our model. In sections 5 and 6, we apply the Structural GARCH model to two applications: asymmetric volatility in equity returns and systemic risk measurement. Finally, Section 7 concludes with suggestions of more applications of the Structural GARCH model.

\section{Structural GARCH}

Our goal is to explore the relationship between the leverage of a firm and its equity volatility. A simple framework to explore this relationship is the classical Merton (1974) model of credit risk and extensions of this seminal work. Equity holders are entitled to the assets of the firm that exceed the outstanding debt. As Merton observed, equity can then be viewed as a call option on the total assets of a firm with the strike of the option being the debt level of the firm. In this model, the fact that firms have outstanding debt of varying maturities is ignored, and we will also adopt this assumption for the sake of maintaining a simple econometric model. The purpose of using structural models is to provide economic intuition for how leverage and equity volatility should interact. It is worth emphasizing that we call our volatility model a "Structural GARCH" because it is motivated from this analysis, not because it derives precisely from a particular option pricing model. In fact, one advantage of our approach is our ability to remain relatively neutral about the true option pricing model that underlies the data generating process.

\subsection{Motivating the Econometric Model}

As is standard in structural models of credit, the equity value of a firm is a function of the asset process and the debt level of the firm. We can therefore define the equity value as 
follows:

$$
E_{t}=f\left(A_{t}, D_{t}, \sigma_{A, t}, \tau, r_{t}\right)
$$

where $f(\cdot)$ is an unspecified call option function, $A_{t}$ is the current market value of assets, $D_{t}$ is the current book value of outstanding debt, $\sigma_{A, t}$ is the (potentially stochastic) volatility of the assets. $\tau$ is the life of the debt, and finally, $r_{t}$ is the annualized risk-free rate at time $t{ }^{6}$ Next, we specify the following generic process for assets and variance:

$$
\begin{aligned}
\frac{d A_{t}}{A_{t}} & =\mu_{A}(t) d t+\sigma_{A, t} d B_{A}(t) \\
d \sigma_{A, t}^{2} & =\mu_{v}\left(t, \sigma_{A, t}\right) d t+\sigma_{v}\left(t, \sigma_{A, t}\right) d B_{v}(t)
\end{aligned}
$$

where $d B_{A}(t)$ is a standard Brownian motion. $\sigma_{A, t}$ captures potential time-varying asset volatility, which we will model formally in Section $2.4^{7}$ The process we specify for asset volatility is general enough to capture popular stochastic volatility models, such as Heston (1993) or the Ornstein-Uhlenbeck process employed by, for example, Stein and Stein (1991). We allow an arbitrary instantaneous correlation of $\rho_{t}$ between the shock to asset returns, $d B_{A}(t)$, and the shock to asset volatility, $d B_{v}(t)$. The specification in Equation (2) encompasses a wide range of stochastic volatility models popular in the option pricing literature. $^{8}$

\footnotetext{
${ }^{6}$ In Appendix A, we re-derive all of the subsequent results in the presence of asset jumps. Because we find the results to be essentially unchanged, we focus on the simpler case of only stochastic volatility for the sake of brevity.

${ }^{7}$ Implicit is that the volatility process satisfies the usual restrictions necessary to apply Itō's Lemma.

${ }^{8} \mathrm{~A}$ short and certainly incomplete list includes Black and Scholes (1973), Heston (1993), and Bates (1996).
} 
The instantaneous return on equity is computed via simple application of Itō's Lemma:

$$
\begin{aligned}
\frac{d E_{t}}{E_{t}}= & \Delta_{t} \frac{A_{t}}{D_{t}} \frac{D_{t}}{E_{t}} \cdot \frac{d A_{t}}{A_{t}}+\frac{\nu_{t}}{E_{t}} \cdot d \sigma_{A, t} \\
& +\frac{1}{2 E_{t}}\left[\frac{\partial^{2} f}{\partial A_{t}^{2}} d\langle A\rangle_{t}+\frac{\partial^{2} f}{d\left(\sigma_{A, t}\right)^{2}} d\left\langle\sigma_{A}^{f}\right\rangle_{t}+\frac{\partial^{2} f}{\partial A \partial \sigma_{A, t}} d\left\langle A, \sigma_{A}\right\rangle_{t}\right]
\end{aligned}
$$

where $\Delta_{t}=\partial f / \partial A_{t}$ is the "delta" in option pricing, $\nu_{t}=\partial f / \partial \sigma_{A, t}$ is the "vega" of the option, and $\langle X\rangle_{t}$ denotes the quadratic variation process for an arbitrary stochastic process $X_{t}$. Here we have ignored the sensitivity of the option value to the maturity of the debt. ${ }^{9}$ In our applications, $\tau$ will be large enough that this assumption is innocuous. All the quadratic variation terms are of the order $\mathcal{O}(d t)$ and we collapse them to an unspecified function $q\left(A_{t}, \sigma_{A, t} ; f\right)$, where the notation captures the dependence of the higher order Itō terms on the partial derivatives of the call option pricing function.

In reality, we do not observe $A_{t}$ because it is the market value of assets. However, given that the call option pricing function is monotonically increasing in its first argument, it is safe to assume that $f(\cdot)$ is invertible with respect to this argument. We further assume that the call pricing function is homogenous of degree one in its first two arguments, which is a standard assumption in the option pricing literature. We define the inverse call option formula as follows:

$$
\begin{aligned}
\frac{A_{t}}{D_{t}} & =g\left(E_{t} / D_{t}, 1, \sigma_{A, t}, \tau, r_{t}\right) \\
& \equiv f^{-1}\left(E_{t} / D_{t}, 1, \sigma_{A, t}, \tau, r_{t}\right)
\end{aligned}
$$

\footnotetext{
${ }^{9}$ For simplicity, we also ignore sensitivity to the risk-free rate, which is trivially satisfied if we assume a constant term structure.
} 
Equation (3) reduces returns to the following: ${ }^{10}$

$$
\begin{aligned}
\frac{d E_{t}}{E_{t}} & =\overbrace{\Delta_{t} \cdot g\left(E_{t} / D_{t}, 1, \sigma_{A, t}^{f}, \tau, r_{t}\right) \cdot \frac{D_{t}}{E_{t}}}^{\equiv L M\left(E_{t} / D_{t}, 1, \sigma_{A, t}, \tau, r_{t}\right)} \frac{d A_{t}}{A_{t}}+\frac{\nu_{t}}{E_{t}} \cdot d \sigma_{A, t}+q\left(A_{t}, \sigma_{A, t}^{f} ; f\right) d t \\
& =\operatorname{LM}\left(E_{t} / D_{t}, 1, \sigma_{A, t}, \tau, r_{t}\right) \times \frac{d A_{t}}{A_{t}}+\frac{\nu_{t}}{E_{t}} \cdot d \sigma_{A, t}+q\left(A_{t}, \sigma_{A, t}^{f} ; f\right) d t
\end{aligned}
$$

For reasons that will become clear shortly, we call $L M\left(E_{t} / D_{t}, 1, \sigma_{A, t}^{f}, \tau, r_{t}\right)$ the "leverage multiplier." When it is obvious, we will drop the functional dependence of the leverage multiplier on leverage, etc., and instead denote it simply by $L M_{t}$. In order to obtain a complete law of motion for equity, we need to know the dynamics of volatility, $\sigma_{A, t}$, as opposed to variance. Itō's Lemma implies that the volatility process behaves as follows:

$$
\begin{aligned}
d \sigma_{A, t} & =\overbrace{\left[\frac{\mu_{v}\left(t, v_{t}\right)}{2 \sigma_{A, t}}-\frac{\sigma_{v}^{2}\left(t, v_{t}\right)}{8 \sigma_{A, t}^{3}}\right]}^{\equiv s\left(\sigma_{A, t} ; \mu_{v}, \sigma_{v}\right)} d t+\frac{\sigma_{v}\left(t, v_{t}\right)}{2 \sigma_{A, t}} d B_{v}(t) \\
& =s\left(\sigma_{A, t} ; \mu_{v}, \sigma_{v}\right) d t+\frac{\sigma_{v}\left(t, v_{t}\right)}{2 \sigma_{A, t}} d B_{v}(t)
\end{aligned}
$$

Plugging Equations (2) and (6) into Equation (5) yields the desired full equation of motion for equity returns:

$$
\begin{aligned}
\frac{d E_{t}}{E_{t}}= & {\left[L M_{t} \mu_{A}(t)+s\left(\sigma_{A, t} ; \mu_{v}, \sigma_{v}\right)+q\left(A_{t}, \sigma_{A, t} ; f\right)\right] d t } \\
& +L M_{t} \sigma_{A, t} d B_{A}(t)+\frac{\nu_{t}}{E_{t}} \frac{\sigma_{v}\left(t, \sigma_{A, t}\right)}{2 \sigma_{A, t}} d B_{v}(t)
\end{aligned}
$$

\footnotetext{
${ }^{10}$ Using the fact that $f(\cdot)$ is homogenous of degree 1 in its first argument also implies that:
}

$$
\Delta_{t}=\partial f\left(A_{t}, D_{t}, \sigma_{A, t}, \tau, r\right) / \partial A_{t}=\partial f\left(A_{t} / D_{t}, 1, \sigma_{A, t}, \tau, r\right) / \partial\left(A_{t} / D_{t}\right)
$$

So with an inverse option pricing formula, $g(\cdot)$ in hand we can define the delta in terms of leverage $E_{t} / D_{t}$. 
Because our empirical focus will be on daily equity and asset returns, we ignore the drift term for equity. Typical daily equity returns are virtually zero on average, so for our purposes ignoring the equity drift is harmless. ${ }^{11}$ Instantaneous equity returns then naturally derive from Equation (7) with no drift:

$$
\frac{d E_{t}}{E_{t}}=L M_{t} \sigma_{A, t} d B_{A}(t)+\frac{\nu_{t}}{E_{t}} \frac{\sigma_{v}\left(t, \sigma_{A, t}\right)}{2 \sigma_{A, t}} d B_{v}(t)
$$

Suppose for a moment that we can ignore the contribution of asset volatility shocks, $d B_{v}(t)$, to equity returns.

Assumption 1. For the purposes of daily equity return dynamics, we can ignore the following term in Equation (8):

$$
\frac{\nu_{t}}{E_{t}} \frac{\sigma_{v}\left(t, \sigma_{A, t}\right)}{2 \sigma_{A, t}} d B_{v}(t)
$$

In Appendix A, we show that Assumption 1 is appropriate in a variety of option pricing models. ${ }^{12}$ The intuition behind this result is as follows: mean reversion is embedded in any reasonable model of volatility. In this case, the time it takes volatility to mean revert is much shorter than typical debt maturities for firms. Thus, the cumulative asset volatility over the life of the option (equity) is effectively constant. In turn, the $\nu_{t}$ term is nearly zero, and so shocks to asset volatility get washed out as far as equity returns are concerned. In the Black-Scholes-Merton (BSM) case, this assumption holds exactly because asset volatility is constant. Under Assumption 1, equity returns and instantaneous equity volatility are given

\footnotetext{
${ }^{11}$ Indeed, ignoring the drift when thinking about long-horizon asset returns (and levels) is not trivial.

${ }^{12}$ That is, when the underlying asset process has jumps, stochastic volatility, stochastic volatility and jumps, etc.
} 
by:

$$
\begin{aligned}
\frac{d E_{t}}{E_{t}} & =L M_{t} \sigma_{A, t} d B_{A}(t) \\
\operatorname{vol}_{t}\left(\frac{d E_{t}}{E_{t}}\right) & =L M_{t} \times \sigma_{A, t}
\end{aligned}
$$

Equation (9) is our key relationship of interest. The equation states that equity volatility (returns) is a scaled function of asset volatility (returns), where the function depends on

financial leverage, $D_{t} / E_{t}$, as well as asset volatility over the life of the option (and the interest rate). The moniker of the "leverage multiplier" should be clear now: $L M_{t}$ describes how equity volatility is amplified by financial leverage. To provide some additional economic intuition about the behavior of $L M_{t}$, we now turn to exploring the shape of the leverage multiplier in some specific settings, and the Black-Scholes-Merton model is a very natural place to start.

\subsection{The Shape of the Leverage Multiplier}

\subsubsection{Leverage Multiplier in the Black-Scholes-Merton World}

It is straightforward to compute $\operatorname{LM}(\cdot)$ when BSM is the relevant option pricing model. To start, we fix annualized asset volatility to $\sigma_{A}=0.15$, time to maturity of the debt $\tau=5$, and the risk-free rate $r=0.03$. Figure 1 plots the leverage multiplier against financial leverage $\left(D_{t} / E_{t}\right)$ in this case.

From Figure 1, we can see that the leverage multiplier is increasing in leverage. Intuitively, when a firm is more leveraged, its equity option value is further from the money and asset returns exceed equity returns by a larger degree. When leverage is zero $\left(D_{t} / E_{t}=0\right)$, the 
Figure 1: BSM Leverage Multiplier

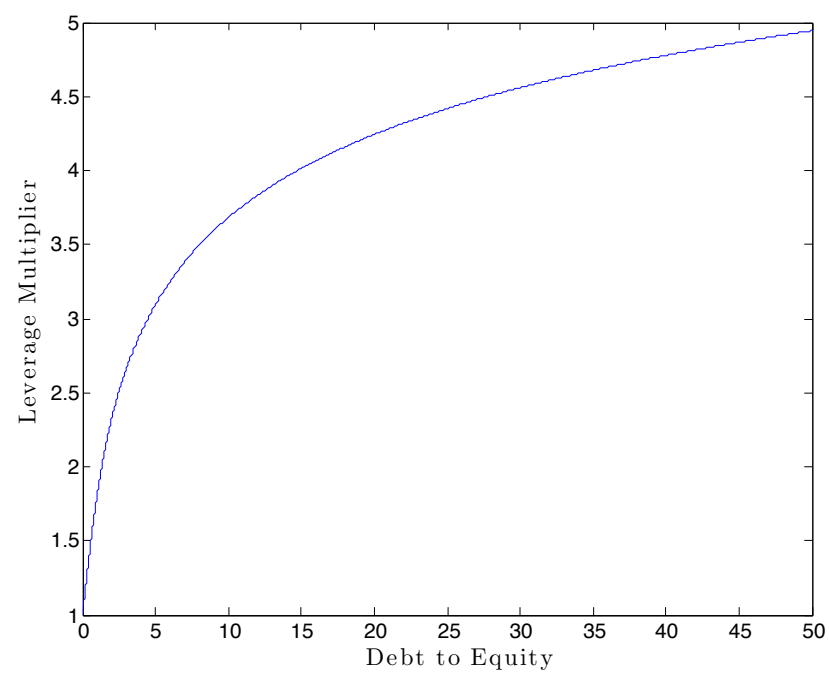

Notes: This figure plots the leverage multiplier in the BSM model. Annualized asset volatility is set to $\sigma_{A}=0.15$, the time to maturity of the debt is $\tau=5$, and the annualized risk-free rate is $r=0.03$.

leverage multiplier is one, because assets must be equal to equity. Next, in Figure 2 we investigate how the BSM leverage multiplier changes as we vary the time to expiration and volatility.

Let us begin with the case where debt maturity is held constant but volatility varies. When volatility increases, the leverage multiplier decreases. In this case, the likelihood that the equity is "in the money" rises with volatility and the effect of leverage on equity volatility is dampened. A similar argument holds when volatility is fixed and debt maturity varies. Extending the maturity of the debt serves to dampen the leverage multiplier because the equity has a better chance of expiring with value. The BSM model provides a useful benchmark in understanding the economics of the leverage multiplier, but it also provides a simple and easy way to compute a set of functions when evaluating $\operatorname{LM}(\cdot)$. Our primary objective is to estimate a simple functional form for $L M(\cdot)$ that is not restricted to the assumptions of the BSM. However, we will ultimately be able to use the functions provided 
Figure 2: BSM Leverage Multiplier with Varying $\sigma_{A}$ and $\tau$

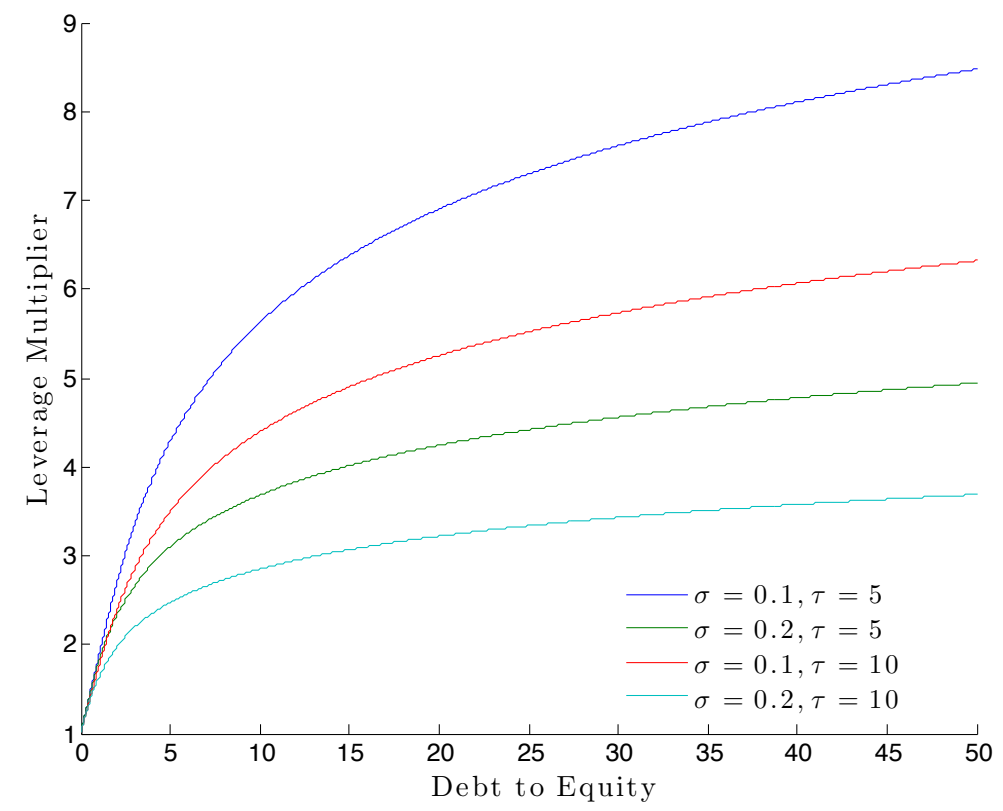

Notes: This figure plots the leverage multiplier in the BSM model. Annualized asset volatility takes on one of two values $\sigma_{A} \in\{0.1,0.2\}$. The time to maturity of the debt also takes on two possible values $\tau \in\{5,10\}$. The annualized risk-free rate is $r=0.03$

by BSM as a starting point for constructing a flexible specification for $L M(\cdot)$.

\subsubsection{The Leverage Multiplier in Other Option Pricing Settings}

The purpose of this subsection is to get a sense of the shape of the leverage multiplier in more complicated option pricing settings. Figure 3 summarizes this analysis visually.

The full details of how we constructed the leverage multiplier in each of the specific option pricing models are found in Appendix A. In addition to the benchmark BSM case, Figure 3 plots the leverage multiplier in the Merton (1976) jump-diffusion model, the Heston (1993) stochastic volatility model, and the stochastic volatility with jumps model employed by Bates (1996) and Bakshi, Cao, and Chen (1997). Figure 3 shows that, for a wide range of leverage, the shape of the leverage multiplier is roughly the same across option pricing models. So 
Figure 3: The Leverage Multiplier in Other Option Pricing Models
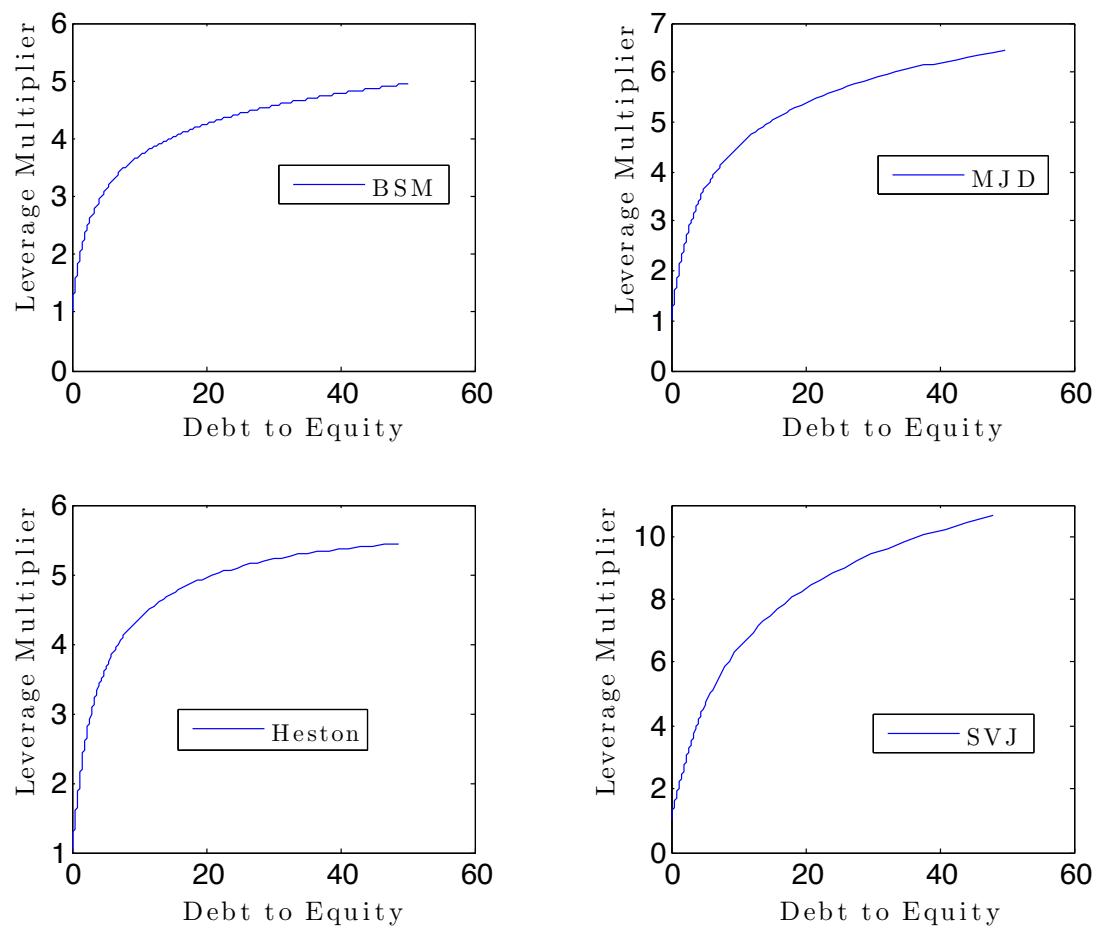

Notes: This figure plots the leverage multiplier in a variety of option pricing models. Full details of the construction can be found in Appendix A. The upper left panel is the benchmark BSM Model. The upper right panel is the Merton (1976) jump-diffusion model. The lower left panel is the Heston (1993) stochastic volatility model. Finally, the lower right panel is a stochastic volatility with jumps model that is used by Bates (1996) and Bakshi, Cao, and Chen (1997).

far, our exploration of the leverage multiplier has been in the context of continuous time. However, our eventual econometric model will fall under the discrete time GARCH class of models for assets. To understand how the leverage multiplier behaves in this setting, we now turn to a Monte Carlo exercise involving GARCH option pricing.

\subsubsection{The Appropriate Leverage Multiplier with GARCH and Non-Normality}

Our Monte Carlo approach is motivated by option models estimated when the underlying follows a GARCH type process, as in Barone-Adesi, Engle, and Mancini (2008). When 
pricing options on GARCH processes, there is often no closed form solution for call prices, necessitating the use of simulation techniques. First, we assume a risk-neutral return process for assets. In our simulations, we adopt four different asset processes: (i) a GARCH(1,1) process with normally distributed innovations; (ii) a $\operatorname{GARCH}(1,1)$ process with $t$-distributed innovations; (iii) an asymmetric $\operatorname{GARCH}(1,1)$ process with normally distributed innovations; and (iv) an asymmetric $\operatorname{GARCH}(1,1)$ process with $t$-distributed errors. The asymmetric GARCH process we use is the GJR process of Glosten, Jagannathan, and Runkle (1993). For completeness, we present these recursive volatility models:

$$
\begin{aligned}
\text { GARCH: } & \sigma_{A, t}^{2}=\omega+\alpha r_{A, t-1}^{2}+\beta \sigma_{A, t-1}^{2} \\
\text { GJR: } & \sigma_{A, t}^{2}=\omega+\alpha r_{A, t-1}^{2}+\gamma r_{A, t-1}^{2} 1_{r_{A, t-1}<0}+\beta \sigma_{A, t-1}^{2}
\end{aligned}
$$

The GJR process captures the familiar pattern in equity returns of negative correlation between volatility and returns; this correlation is captured by the asymmetry parameter, $\gamma$. In our parameterization of these processes, we set the asymmetry parameter to be quite large, because this is one way to capture how risk-aversion affects the risk-neutral asset process. In addition, for the models with $t$-distributed innovations, we set the degrees of freedom to six in order to fatten the tails of the asset return process. In order to ensure comparability across models within our simulation, we change $\omega$ so that the unconditional volatility of all the processes is 15 percent annually. Table 1 summarizes our parametrization. 
Table 1: Parameterizations for Simulated-Asset Processes

\begin{tabular}{|c|c|c|c|}
\hline & \multicolumn{4}{|c|}{ Parameter } \\
Model & $\alpha$ & $\gamma$ & $\beta$ \\
\hline GARCH with Normal Errors & 0.07 & - & 0.92 \\
\hline GARCH with $t$ Errors & 0.07 & - & 0.92 \\
\hline GJR with Normal Errors & 0.022 & 0.18 & 0.884 \\
\hline GJR with $t$ Errors & 0.022 & 0.18 & 0.884 \\
\hline
\end{tabular}

Notes: The table provides parameter values for the volatility models used to generate the leverage multiplier in discrete time environments. We assume assets follow each of the four volatility models in the table above and we then simulate the leverage multiplier according to each parametrization.

For each process, we simulate the asset process 10,000 times from an initial asset value of $A_{0}=1$. We assume the debt matures in two years and, for simplicity, set the risk-free rate to zero. The simulation generates a set of terminal values, $A_{T}$, which in turn generate an equity value for each value of debt $D .{ }^{13}$ We then compute numerical derivatives to measure how the equity value changes with respect to $A_{0}$. Finally, we calculate the leverage multiplier implied by each asset return process and plot it against the implied financial leverage in Figure 4 .

The economics behind the shape of the leverage multiplier under various asset return processes are subtle. The benchmark case of BSM is given by the blue line in Figure 4, and it is easy to see that in a symmetric setting, making the tails of the asset distribution longer via GARCH decreases the leverage multiplier for larger values of debt (the green and red lines). For larger values of debt, extending the tails of the asset distribution serves the same function as increasing volatility in the BSM case. When we introduce asset volatility asymmetry via the GJR process, the leverage multiplier increases dramatically relative to the BSM benchmark (turquoise and purple lines). Volatility asymmetry effectively makes the figure asset distribution left skewed, which shortens the right tail of the distribution and

\footnotetext{
${ }^{13} E=\frac{1}{10,000} \sum_{i=1}^{10,000} \max \left(A_{T, i}-D, 0\right)$, where $i$ is the index for each simulation run. Varying $D$ generates a variable range of leverage, $D / E$.
} 
Figure 4: Simulated Leverage Multiplier in Stochastic Volatility and Non-Normality

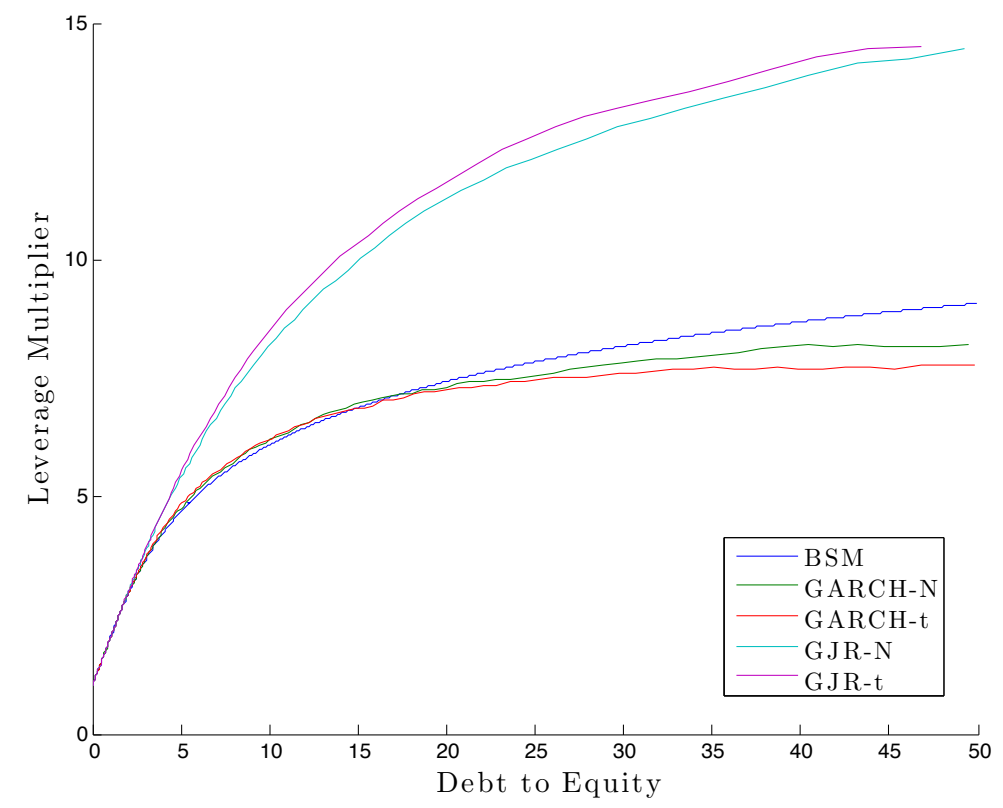

Notes: The figure plots the simulated leverage multiplier under different asset return process specifications. We consider GARCH and GJR process, each with normally distributed and $t$ distributed errors. The unconditional volatility in all the models is 15 percent annually, the time to maturity of the debt is two years, and the risk-free rate is set to zero. The parameters of each volatility model can be found in Table 1.

increases the leverage multiplier. In this case, leverage has a larger amplification on equity volatility because high leverage corresponds to a much smaller likelihood the equity expires "in the money."

\subsubsection{Three Properties of the Leverage Multiplier}

In general, it is clear that the shape of the leverage multiplier is robust across a variety of continuous time and discrete time option pricing models. More specifically, our preceding analysis implies that the leverage multiplier satisfies at least three basic properties: (i) when leverage is zero, the leverage multiplier has a value of one, (ii) the leverage multiplier is weakly increasing in leverage, and (iii) the leverage multiplier is concave in leverage. 
As previously discussed, the first property is mechanical and true by definition. It is slightly easier to prove the latter two properties within a specific option pricing framework, though it has proven more difficult to do so in a general setting. However, because we have shown that the leverage multiplier satisfies these three properties in a number of different option pricing models, we believe these three properties are not model dependent and likely derive from no arbitrage arguments. Perhaps more mildly, these properties should apply to asset processes whose distributions are plausible in the real world (that is, not a degenerative risk-neutral distribution with all the mass at some extreme point) and for reasonable levels of leverage. The remainder of our analysis will take these three properties as given. With this in mind, we propose a parameterized function to capture leverage amplification mechanisms in a relatively "model-free" way.

\subsection{A Flexible Leverage Multiplier}

In the derivation of Equation (9), we did not assign specific functions to $g(\cdot)$ and $\Delta_{t}$. We define $g^{B S M}(\cdot)$ and $\Delta_{t}^{B S M}$ as the BSM inverse call and delta functions. We then propose the following specification for the leverage multiplier:

$\operatorname{LM}\left(D_{t} / E_{t}, \sigma_{A, t}^{f}, \tau, r_{t} ; \phi\right)=\left[\Delta_{t}^{B S M}\left(E_{t} / D_{t}, 1, \sigma_{A, t}^{f}, \tau, r_{t}\right) \times g^{B S M}\left(E_{t} / D_{t}, 1, \sigma_{A, t}^{f}, \tau, r_{t}\right) \times \frac{D_{t}}{E_{t}}\right]^{\phi}$

In this case, $\phi$ is the departure from the BSM model. When taking our model to data, it will be an estimated parameter. One advantage of our proposed leverage multiplier in Equation (10) is its relative simplicity in terms of computation, as the BSM delta and inverse call functions are numerically tractable. We discuss these potential computation issues later in Section 3.2. 
Figure 5: Leverage Multiplier for Different Values $\phi$

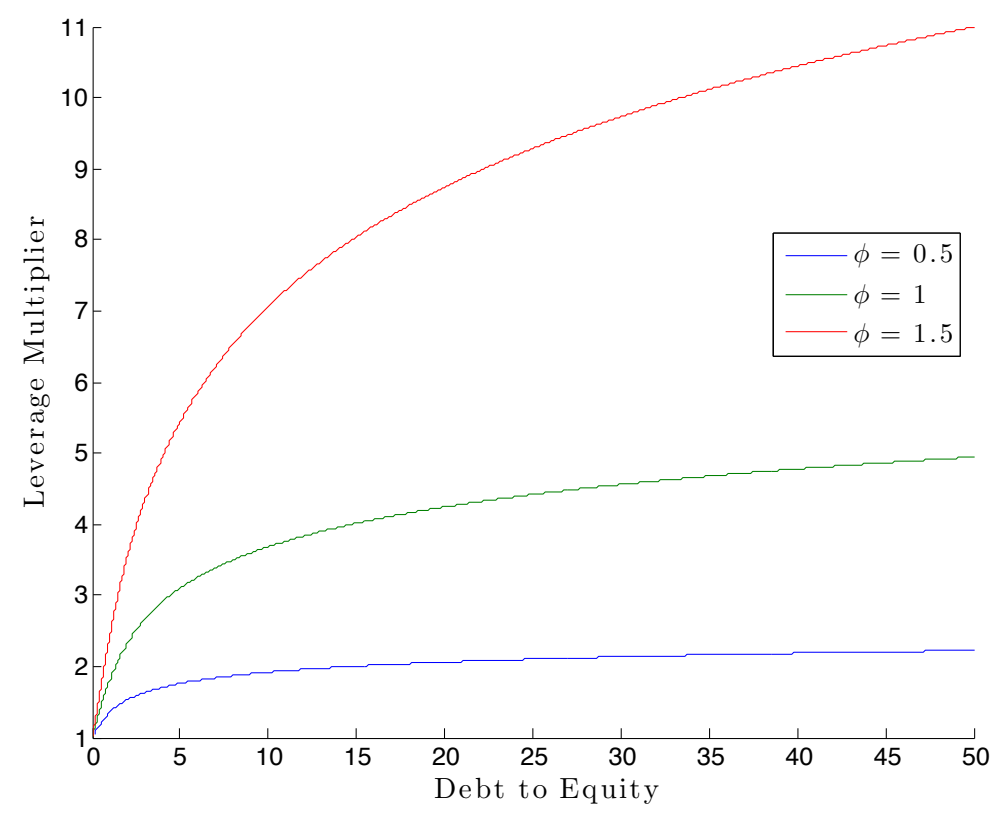

Notes: This figure plots the leverage multiplier according to the specification in (10) for different values of $\phi$. In our baseline case, the annualized $\sigma_{A}$ is held constant at $0.15, \tau=5$, and $r=0.03$.

It is worth emphasizing that our leverage multiplier simply uses a mathematical transformation of the BSM functions. For example, in a BSM world, $g^{B S M}(\cdot)$ would be interpreted as the asset-to-debt ratio, but for our model it is simply a function. Similarly, $\Delta_{t}^{B S M}$ in our specification is not interpreted as the correct hedge ratio, but merely serves as a function for our purposes. Let us now examine how our leverage multiplier changes for different values of $\phi$, which we plot in Figure 5.

Unsurprisingly, increasing $\phi$ increases the leverage multiplier. For firms with a low value of $\phi$, high levels of leverage have a small amplification effect in terms of equity volatility. Building on the intuition from the BSM case, we see that for these firms leverage plays a small role in the moneyness of the equity, which likely corresponds to healthier firms. The converse holds true as well, as firms with high $\phi$ experience large equity volatility amplification, even 
Figure 6: Simulated Leverage Multiplier and Our Specification

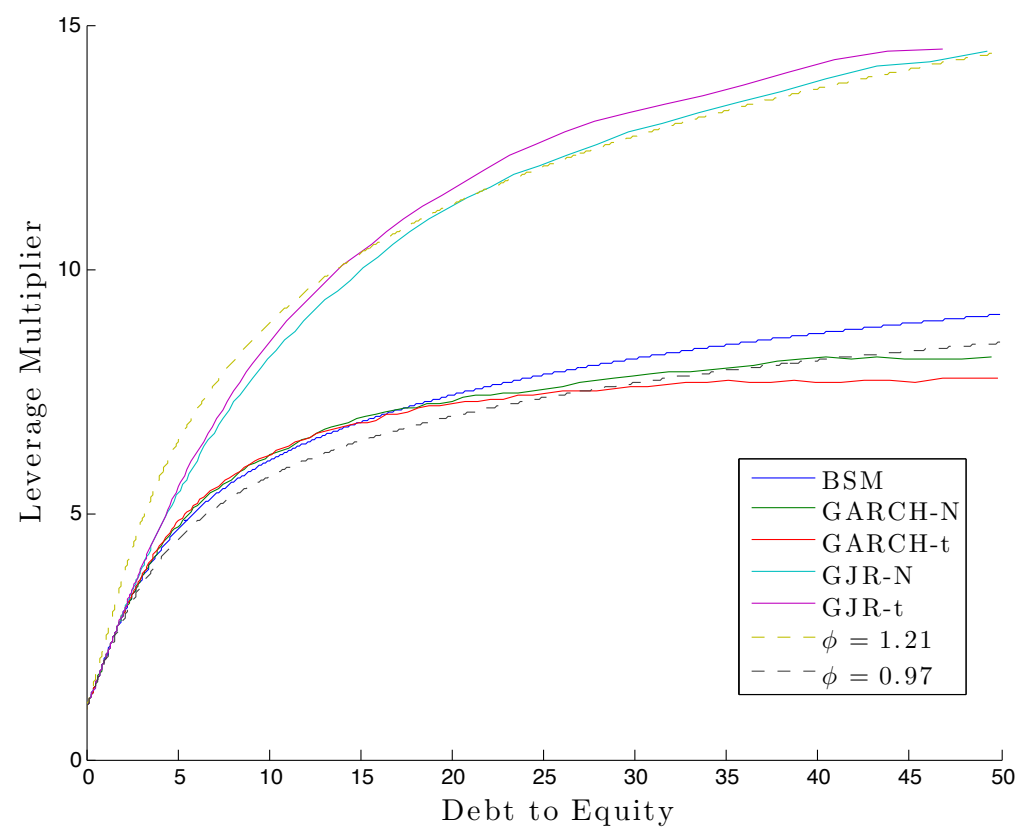

Notes: The figure plots the simulated leverage multiplier under different asset return process specifications. We consider GARCH and GJR process, each with normally distributed and $t$ distributed errors. The unconditional volatility in all the models is 15 percent annually, the time to maturity of the debt is two years, and the risk-free rate is set to zero. The parameters of each volatility model can be found in Table 1. In addition, we plot our leverage multiplier from specification (10) for different values of $\phi$ to demonstrate that our model captures various asset return processes well.

for low levels of financial leverage.

To highlight the flexibility of our specification, we revisit the Monte Carlo exercise from Section 2.2.3. Figure 6 plots the leverage multiplier in a GARCH option pricing setting, as well as our leverage multiplier for a few different values of $\phi$.

Figure 6 shows that varying $\phi$ in our leverage multiplier specification captures various asset return processes well. Increasing $\phi$ is successful in matching the patterns in the leverage multiplier that arise in stochastic volatility, asymmetric volatility, and non-normal settings.

Importantly, our flexible leverage multiplier also preserves the three necessary properties outlined in Section 2.2.4. Raising the BSM leverage multiplier to an arbitrary power naturally 
preserves the condition for $L M(\cdot)$ to have a value of one when leverage is zero. It is also clear from Figure 5 and Figure 6 that varying $\phi$ preserves the concavity and increasing nature of the BSM leverage multiplier. ${ }^{14}$ While our specification is seemingly simple, it is not a trivial task to define a function that retains the flexibility of ours but also maintains the necessary properties of the leverage multiplier. Our analysis in Section 2.2.1 also demonstrated that $L M$ is decreasing in asset volatility and time-to-maturity. ${ }^{15}$ Because our leverage multiplier is a power function of the BSM multiplier, an additional advantage of our specification is that it inherits these natural properties from the Black-Scholes-Merton model.

\subsection{The Full Recursive Model}

The preceding analysis motivates the use of our leverage multiplier in describing the relationship between equity volatility and leverage. To make the model fully operational in discrete time, we propose the following process for equity returns:

$$
\begin{aligned}
r_{E, t} & =L M_{t-1} r_{A, t} \\
r_{A, t} & =\sqrt{h_{A, t}} \varepsilon_{A, t}, \quad \varepsilon_{A, t} \sim D(0,1) \\
h_{A, t} & =\omega+\alpha\left(\frac{r_{E, t-1}}{L M_{t-2}}\right)^{2}+\gamma\left(\frac{r_{E, t-1}}{L M_{t-2}}\right)^{2} 1_{r_{E, t-1}<0}+\beta h_{A, t-1} \\
L M_{t-1} & =\left[\triangle_{t-1}^{B S M} \times g^{B S M}\left(E_{t-1} / D_{t-1}, 1, \sigma_{A, t-1}^{f}, \tau\right) \times \frac{D_{t-1}}{E_{t-1}}\right]^{\phi}
\end{aligned}
$$

\footnotetext{
${ }^{14}$ To be precise, $\phi$ preserves the concavity so long as it is not too large, long-run asset volatility is not too small, and $\tau$ is not too small. In practice, this is not an issue, even for financial firms who have larger amounts of leverage. When we estimate the model, we later verify that none of the fitted $\phi$ result in violations of this sort.

${ }^{15}$ Our analysis in Section 2.2.1 applied to the BSM model, but the notion that the leverage multiplier is decreasing in both asset volatility and time to maturity holds more broadly. Merton (1974) shows that as the time-to-maturity goes to infinity, the option becomes the same as the underlying, so the leverage multiplier must decrease to its lower bound of one (Theorem 3). Similarly, the call option pricing formula is weakly increasing in volatility (Theorem 8). So long as the rate of increase in the delta of the option w.r.t volatility is slower than for the underlying option price, the leverage multiplier will be decreasing in asset volatility.
} 
We will call the specification described in Equation (11) as a "Structural GARCH" model. ${ }^{16}$ The parameter set for the Structural GARCH is $\Theta:=(\omega, \alpha, \gamma, \beta, \phi)$, so there is only one extra parameter compared to a vanilla GJR model. We will confront the issue of how to compute $\tau$ and $\sigma_{A, t-1}^{f}$ in the next section when describing the data and estimation techniques used in our empirical work. We also introduce lags in the appropriate variables (e.g., the leverage multiplier) to ensure that one-step ahead volatility forecasts are indeed in the previous day's information set. The model in (11) nests both a simple GJR model $(\phi=0)$ and the BSM model $(\phi=1)$, and provides a statistical test of how leverage affects equity volatility. ${ }^{17}$ This is another attractive feature of our leverage multiplier from an econometric perspective, and adds to the theoretically appealing qualities we highlighted in Section 2.3.

The equity return series will inherit volatility asymmetry from the asset return series, an important feature of equity returns in the data. ${ }^{18}$ The recursion for equity returns (and asset returns) in (11) is simple and straightforward to compute, yet powerful. For example, when simulating this model, if a series of negative asset returns is realized (and hence negative equity returns since they share the same shock), volatility rises due to the asymmetric specification inherent in the GJR. In that case, leverage also rises, increasing the leverage multiplier and resulting in an even stronger amplification effect for equity volatility. As we

\footnotetext{
${ }^{16}$ In reality our model is a Structural GARCH $(1,1)$ model, because it includes a single lag of the squared asset return and asset volatility. Incorporating a richer lag structure is straightforward, so that our model can naturally be generalized to $\operatorname{Structural} \operatorname{GARCH}(p, q)$ model as follows:

$$
h_{A, t}=\omega+\sum_{j=1}^{p} \alpha_{j}\left(\frac{r_{E, t-j}}{L M_{t-j-1}}\right)^{2}+\sum_{j=1}^{p} \gamma_{j}\left(\frac{r_{E, t-j}}{L M_{t-j-1}}\right)^{2} 1_{r_{E, t-j}<0}+\sum_{i=1}^{q} \beta_{i} h_{A, t-i}
$$

${ }^{17} \phi=1$ nests the BSM exactly if we use a constant forecast of asset volatility over the lifetime of the option. As mentioned, we estimate this model against a model where we use a GJR forecast for $\sigma_{A, t}^{f}$. The results are similar, so we refer to the two without distinction.

${ }^{18}$ For example, it is has been shown that a GJR process for equity can replicate features of equity option data like the volatility smirk.
} 
saw in the recent financial crisis, this was a key feature of the data, particularly for highly leverage financial firms. Additionally, by letting $\phi$ vary from firm-to-firm, we effectively allow a different option pricing model to apply to the capital structure of each firm. This flexibility is difficult to achieve if we impose an option pricing model on the data a priori because, as we showed, $\phi$ allows us to move across different classes of option pricing models. To the extent that our leverage multiplier form captures various option pricing models, the Structural GARCH allows us to infer a high frequency asset return series with stochastic volatility in a relatively model-free way. Later, this will prove to be extremely useful for a number of applications of the model.

We also wish to emphasize there are many ways to parameterize the observation that the leverage multiplier is similar across option pricing models for the purposes of volatility mod-

eling. We have chosen a particular specification that balances parsimony with the underlying economics, while still retaining useful statistical properties. However, the themes that underlie the Structural GARCH are broader than our specific econometric model. An additional contribution of this paper is to provide a simple and economically grounded framework with widespread application for modeling volatility and leverage jointly.

\section{Data Description and Estimation Details}

\subsection{Data Description}

We now turn to estimating the Structural GARCH model using equity return data. To compute the leverage multiplier, we also need balance sheet information. Unless otherwise noted, we obtain all of our data from Bloomberg. In particular, we define $D_{t}$ as the book 
value of debt at time $t .^{19}$ To avoid estimation issues inherent with quarterly data, we smooth the book value of debt using an exponential average with smoothing parameter of 0.01 . This smoothing parameter value implies a half-life of approximately 70 days in terms of the weights of the exponential average, which is reasonable for quarterly data.

The set of firms we analyze are financial firms over a period that spans from January 3, 1990 to February 14, 2014. ${ }^{20}$ The reasons we focus on financial firms are twofold: first, these firms typically have extraordinarily high leverage and structural models have failed to model these firms well. Second, given the high volatility in the recent crisis that was accompanied by unprecedented leverage, this set of firms presents an important sector to model from a systemic risk and policy perspective. To this end, one of the applications of our model that we will explore in later sections involves systemic risk measurement of financials. In future work, we hope to extend the set of firms we analyze.

\subsection{Numerical Implementation}

When estimating the full model, we use quasi-maximum likelihood and the associated standard errors for parameter estimates. In order to ensure a global optimum is reached, we also conduct each maximum likelihood optimization over a grid of 24 different starting values. $^{21}$ Despite the relative simplicity of our model, quasi-maximum likelihood estimation is still quite costly from a computational perspective. To see why, let us explicitly define our

\footnotetext{
${ }^{19}$ All liabilities are treated as exogenous and as if they have a single expiration. Liabilities are measured as the book value of assets minus the book value of equity from quarterly accounting statements. Some models use maturity measures directly however for financial firms many of the liabilities do not have contractual liabilities. We discuss the issue of debt maturity below.

${ }^{20} \mathrm{~A}$ full description of the set of firms is contained in Appendix D.1.

${ }^{21}$ The Matlab code for estimation of the model via quasi-maximum likelihood (QMLE) with the correct standard errors is available upon request.
} 
log-likelihood function from the specification in (11):

$$
\begin{aligned}
\mathcal{L}\left(\omega, \alpha, \gamma, \beta, \phi ;\left\{r_{E, t}, E_{t}, D_{t}\right\}_{t=2}^{T}\right) & :=-\frac{1}{2} \sum_{t=2}^{T}\left[\log (2 \pi)+\log \left(h_{E, t}\right)+\frac{\left(r_{E, t}\right)^{2}}{h_{E, t}}\right] \\
& =-\frac{1}{2} \sum_{t=2}^{T}\left[\log (2 \pi)+\log \left(L M_{t-1}^{2} h_{A, t}\right)+\frac{\left(r_{E, t}\right)^{2}}{L M_{t-1}^{2} h_{A, t}}\right]
\end{aligned}
$$

where all summations begin from $t=2$ because the leverage multiplier contains lagged equity and debt values. From our definition of the leverage multiplier, it is clear that a single computation of $L M_{t}$ requires an inversion of the BSM call option formula. For a firm with 10 years of data, this means evaluating $\mathcal{L}(\cdot)$ at a single parameter set requires approximately $10 \times 252=2520$ inversions of the BSM call option formula. In turn, maximizing a single firm's likelihood function typically involves 180 function evaluations, which means $2520 \times 180=$ 453, 600 inversions. As mentioned, we use 24 different starting values to ensure a global maximum is reached, two different types of asset volatility over the life of the debt, and 30 different debt maturities (more details follow). In total, this means for an average firm in our sample, we must invert the BSM function $2520 \times 280 \times 24 \times 2 \times 30=1,016,064,000$ times, which is computationally expensive given there is no closed form formula for the inverse BSM function. ${ }^{22}$

To make the problem computational tractable, we estimate all of our models on the Amazon Elastic Compute Cloud. The computing unit we use is their latest generation Linux based machine with $32 \mathrm{CPUs}$, and $60 \mathrm{~GB}$ of RAM. Estimation of each firm is done using parallel processing, and the average firm takes about 80 minutes to estimate the full model. Since we estimate the model for more than 80 firms, we use many different computing

\footnotetext{
${ }^{22}$ Later in Section 6, we will simulate the Structural GARCH models thousands of times over long horizons, also a computationally taxing task for similar reasons.
} 
units simultaneously to make the total time more reasonable (approximately 12 hours for all firms).

The remaining issues are how to treat both the time to maturity of the debt $\tau$, the asset volatility over the life of the debt $\sigma_{A, t}^{f}$, and the risk-free rate, $r_{t}$.

\section{Time to Maturity of the Debt}

An input to the leverage multiplier is time to maturity of the debt. Because the book value of debt combines a number of different debt maturities, we simply iterate over different $\tau$ during estimation. Specifically, we estimate the model for $\tau \in[1,30]$, restricting $\tau$ to take on integer values. We keep the version of the model that attains the highest log-likelihood function.

\section{Risk Free Rate}

To compute the leverage multiplier, we must also input the risk free rate over the life of the debt. We do so by using a zero-curve provided by OptionsMetrics, which is derived from BBA LIBOR rates and settlement prices of CME Eurodollar futures. We then linearly interpolate (with flat endpoints beyond the maximum maturity) to determine the riskless rate for a specific maturity.

\section{Asset Volatility Over Life of Debt}

We take two different approaches for the computing the value of $\sigma_{A, t}^{f}$. The first is to use the unconditional volatility implied by the asset volatility series corresponding to the unconditional volatility of a GJR process. Using a constant $\sigma_{A, t}^{f}$ in fact completely eliminates any issues in ignoring the vega terms in our motivating derivation of the leverage multiplier (see Equation (3)). The second approach is to use the GJR forecast over the life of the debt 
at each date $t$. It is straightforward to derive the closed form expression for this forecast. We use both approaches for $\sigma_{A, t}^{f}$ and choose the model with the highest likelihood.

\section{Empirical Results}

\subsection{Cross-Sectional Summary}

We begin by presenting a cross-sectional summary of the estimation results. ${ }^{23}$ Since the main contribution of this paper is the leverage multiplier, Figure 7 plots the estimated time-series of the lower quartile, median, and upper quartile leverage multipliers, across all firms.

As we can see, there is considerable cross-sectional heterogeneity in the leverage multiplier, even within financial firms. It appears that across all firms, the leverage multiplier moves with the business cycle, which is not surprising given that leverage itself tends to do so as well. In the top quartile of firms, leverage amplified equity volatility by a factor of eight during the financial crisis. Evidently, for this set of firms, the leverage amplification mechanism has remained high in the years following the crisis.

Table 1 shows cross-sectional summary statistics for the point estimates of the Structural GARCH model. In our model, the first four estimates represent the GJR parameters for the asset return series. It is not surprising then that they resemble those found in equity returns. The parameter $\omega$ is an order of magnitude smaller than usual, but this is natural because asset returns are less volatile than equity returns and $\omega$ is a determinant of the unconditional volatility. The asset process is indeed stationary, as seen by the combination of $\alpha, \gamma, \beta$ and

\footnotetext{
${ }^{23}$ There were 11 firms where the estimated $\phi$ coefficient had convergence issues and hit the lower bound for $\phi$. We discuss these firms specifically in Appendix D.2. The main unifying theme with these firms is that their leverage is both low and nearly constant through the time series, so identification of $\phi$ is difficult. We exclude these firms for the remainder of the analysis.
} 
Figure 7: Time Series of Leverage Multiplier Across Quartiles

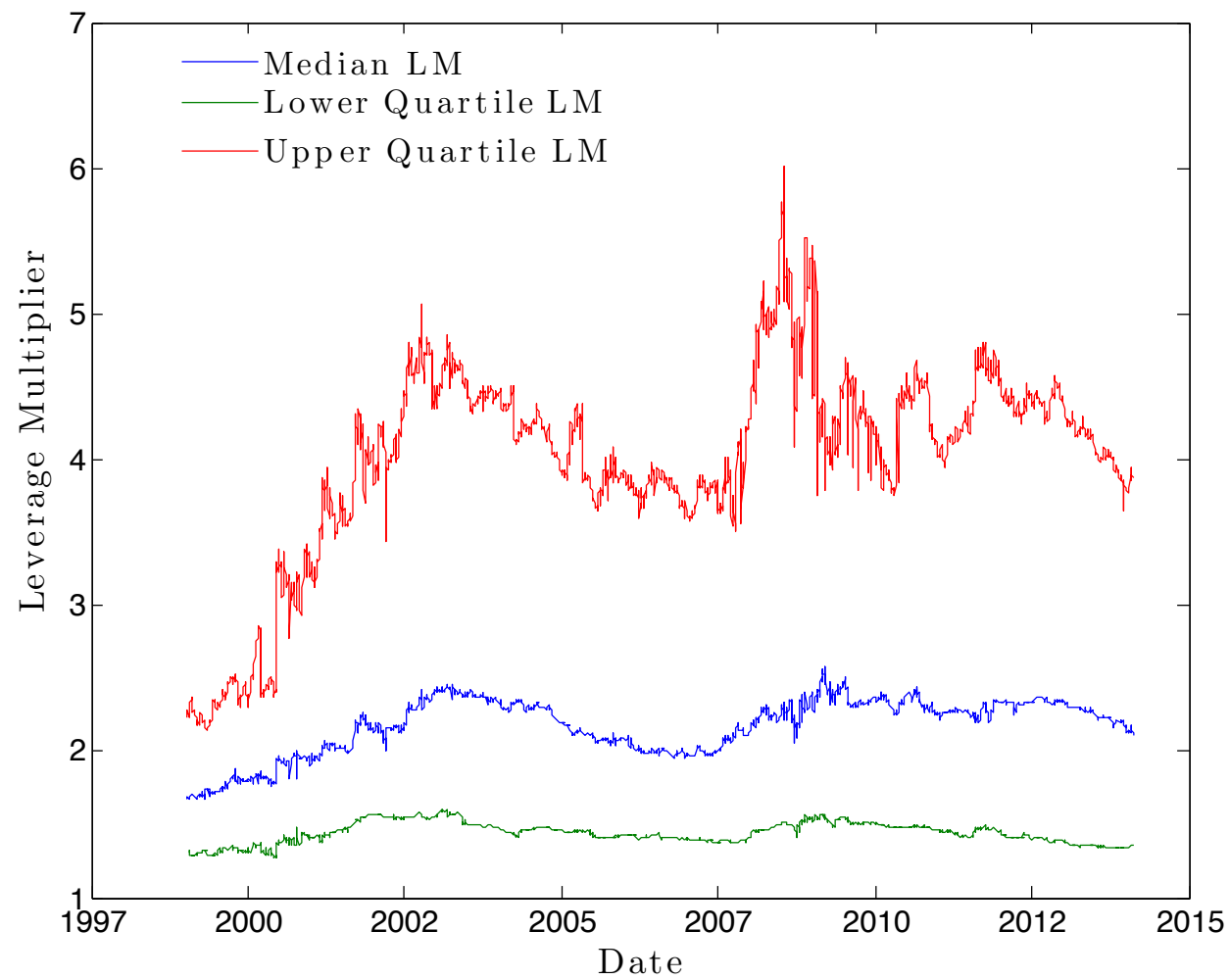

Notes: The figure plots the quartiles of the estimated leverage multiplier across firms, and through time. The plot begins in January 1998 and ends in February 2014. The full set of firms we analyze can be found in Appendix D.1

standard results on the stationarity of GARCH processes. One subtle but key difference in the current estimates is the parameter $\gamma$, which is higher than it is for equity returns in this subset of stocks. Recall that $\gamma$ dictates the correlation between volatility and returns, and thus it appears that the volatility asymmetry we observe in equity is somewhat dampened in asset returns. In one application of the model, we will explore this idea further as it pertains to the classical leverage effect of Black (1976) and Christie (1982).

The new parameter in our model is $\phi$. The third and fourth columns of Table 2 show $\phi$ is statistically different than zero for a majority of firms. Therefore, the effect of leverage on 
Table 2: Cross-Sectional Summary of Structural GARCH Parameter Estimates

\begin{tabular}{|c|c|c|c|}
\hline Parameter & Mean & Mean t-stat & \% with $|t|>1.64$ \\
\hline$\omega$ & $2.7 \mathrm{e}-06$ & 1.70 & 47.2 \\
\hline$\alpha$ & 0.0458 & 3.07 & 86.1 \\
\hline$\gamma$ & 0.0721 & 2.91 & 80.6 \\
\hline$\beta$ & 0.9024 & 80.08 & 100 \\
\hline$\phi$ & 0.9834 & 4.00 & 73.6 \\
\hline
\end{tabular}

Notes: This table provides a cross-sectional summary of the parameter estimates from the Structural GARCH model as defined in Equation (11). The full set of firms we analyze can be found in Appendix D.1.

equity volatility via our leverage multiplier appears to be substantial for a large number of financial firms. Interestingly, the average $\phi$ is slightly less than one, as the BSM model would suggest. These results are roughly consistent with the findings of Schaefer and Strebulaev (2008) who find that while the Merton (1974) model does poorly in predicting the levels of credit spreads, it is successful in generating the correct hedge ratios across the capital structure of the firm. In our context, we interpret their finding and our estimation of $\phi$ to mean that the Merton model does well in recovering the daily returns of assets well, even if it is not able to pinpoint the level of assets.

\subsection{Aggregation}

\subsubsection{Aggregate Leverage Multiplier}

We aggregate our results across firm by creating three indices: 1) a value-weighted average equity volatility index, 2) a value-weighted average asset volatility index, and 3) an aggregate leverage multiplier. The aggregate leverage multiplier is simply the ratio of the equity volatility index to the asset volatility index. The weights used in creating each respective index are derived from equity valuations. Figure 8 plots these three time series. Again, it 
Figure 8: Aggregate Equity Volatility, Asset Volatility, and Leverage Multiplier
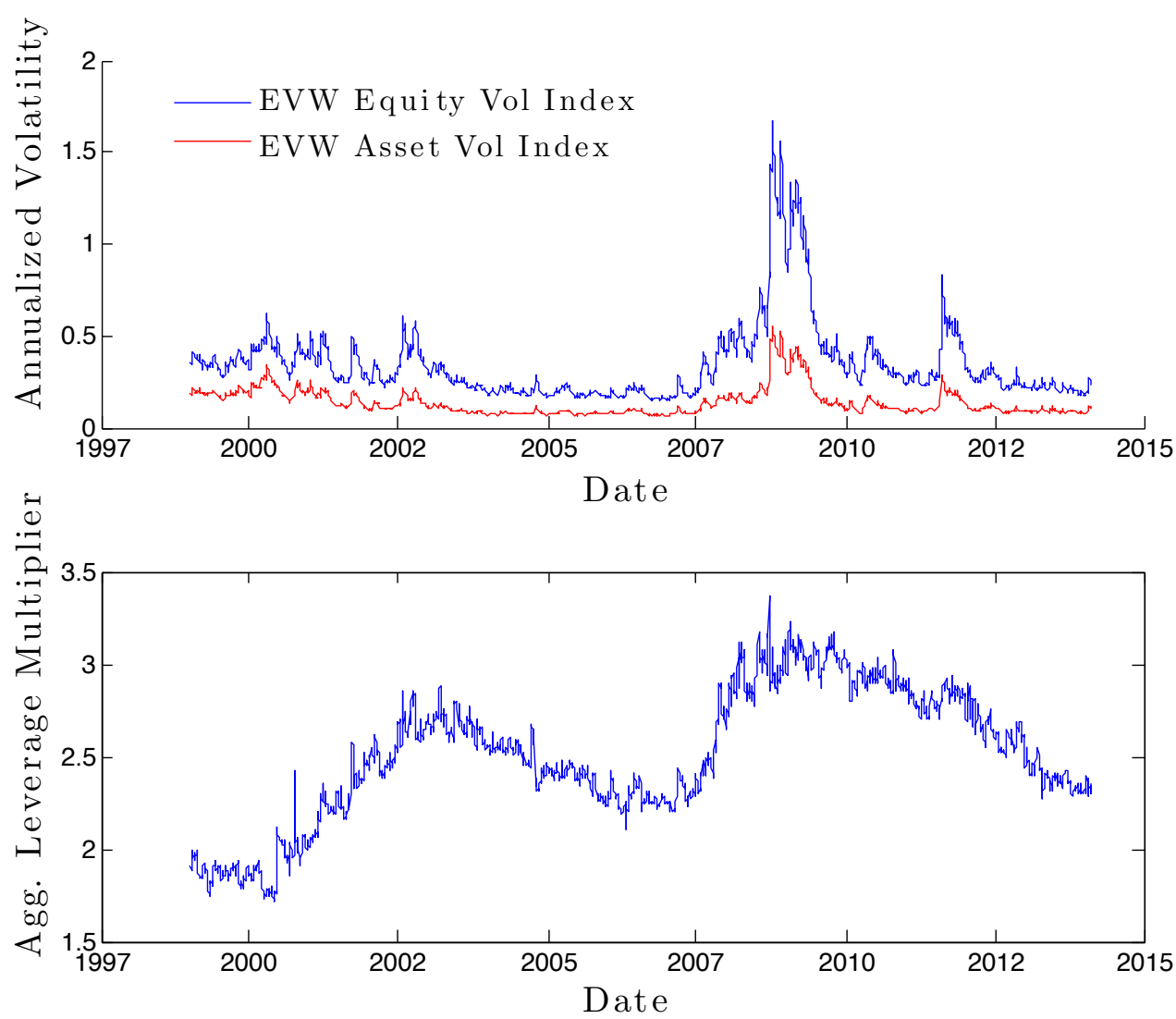

Notes: This figure plots the value-weighted average across all firms of our estimated equity volatility and asset volatility. The weights used in creating each respective index are based on equity valuations. The aggregate leverage multiplier is then the ratio of the aggregate equity volatility index to the asset volatility index.

is clear that there is a cyclicality in the aggregated leverage multiplier. A pressing issue in the wake of the financial crisis is the role of leverage and the health of the financial sector. Since our model provides estimates of leverage amplification in terms of equity volatility (as well as asset volatility), we focus on these aggregated time-series through the financial crisis in Figure 9.

It is clear that the rise in equity volatility for the aggregate financial sector began in the 
Figure 9: Aggregate Equity Volatility, Asset Volatility, and Leverage Multiplier During Financial Crisis
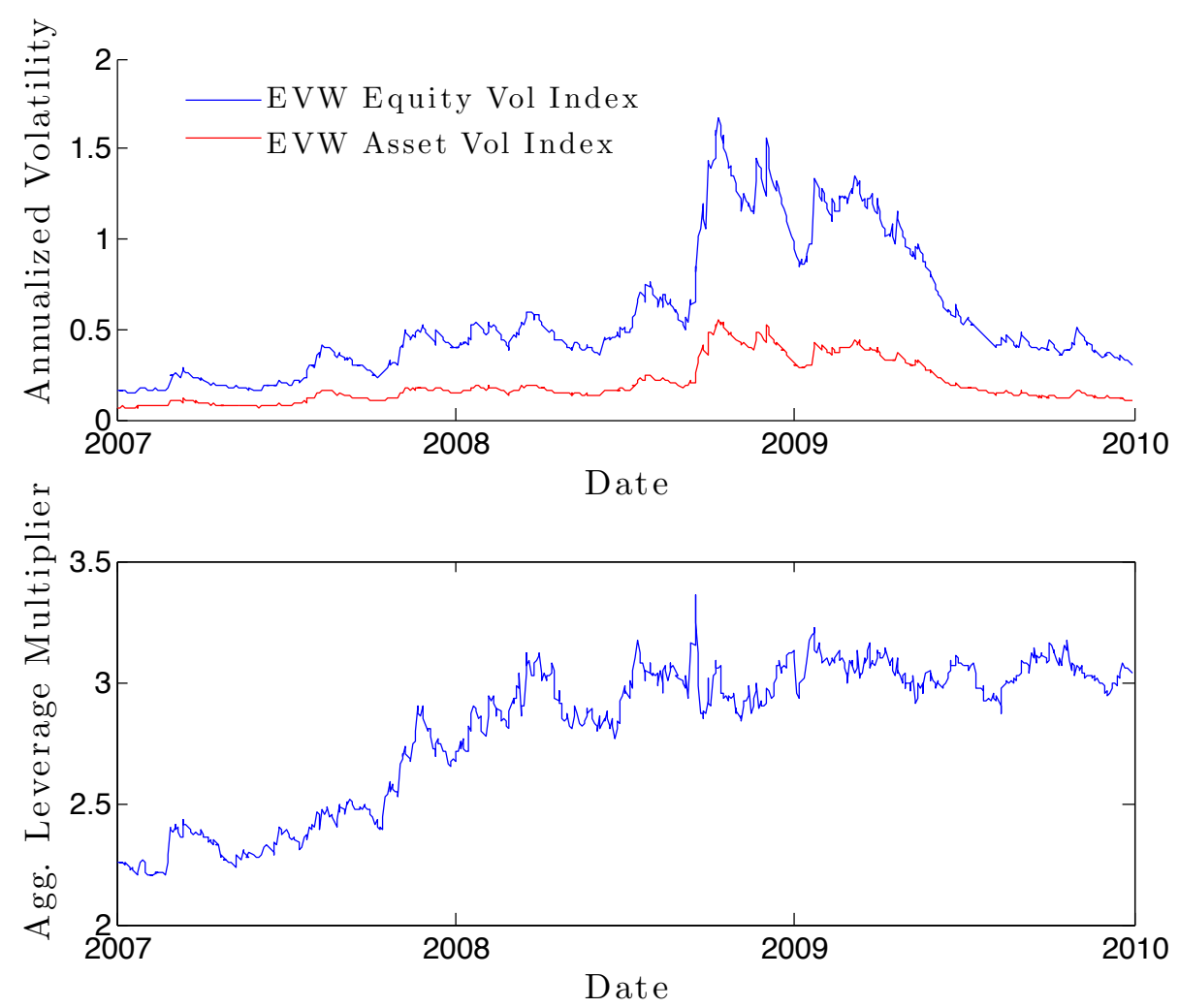

Notes: This figure plots the value-weighted average across all firms of our estimated equity volatility and asset volatility. The weights used in creating each respective index are based on equity valuations. The aggregate leverage multiplier is then the ratio of the aggregate equity volatility index to the asset volatility index. Our time period focuses on the financial crisis from 2007 to mid-2009.

summer of 2007. However the rise in asset volatility did not really occur until late in 2008. The increase in leverage in 2007 was partly an increase in aggregate liabilities and partly a fall in equity valuation. After the fall of Lehman Brothers Holdings, Inc., asset volatility rose dramatically as well and the leverage multiplier continued to rise before stabilizing in the spring of 2009 . 


\section{The Leverage Effect}

We now turn to our first application of the Structural GARCH: the leverage effect. The leverage effect of Black (1976) and Christie (1982) documents the negative correlation that exists between equity returns and equity volatility. One possible explanation for this fact is that when a firm experiences a fall in equity, its financial leverage mechanically rises, the company becomes riskier, and volatility rises.

A second explanation points to the role of risk premiums in describing the negative correlation between equity returns and equity volatility (e.g. French, Schwert, and Stambaugh (1987)). In this explanation, a rise in future volatility raises the required return on equity, leading to an immediate decline in the stock price. The Structural GARCH model provides a natural framework to explore these issues econometrically. ${ }^{24}$

Recall that the Structural GARCH model delivers an estimate of the daily return of assets. $^{25}$ Any correlation between asset volatility and asset returns can not be due to financial leverage. So, if a correlation does exist, it must be attributed to a risk-premium argument. When applying the GJR volatility model to a given time series of returns, the $\gamma$ parameter is one way to measure the correlation between the volatility and returns (e.g. a higher $\gamma$ corresponds to more negative correlation). Therefore, we would expect the GJR $\gamma$ estimated from equity returns to be larger than the same parameter estimated from asset returns. Indeed, the median $\gamma$ for equity returns is 0.0811 and the median $\gamma$ for asset returns is

\footnotetext{
${ }^{24}$ Other econometric studies of the leverage effect include Bekaert and $\mathrm{Wu}$ (2000). The difference in our approach is that we allow debt for the firm to be risky, as in the Merton (1974) model.

${ }^{25}$ Again, this relies on a few assumptions. First, our specification ignores the effect of changes in long-run asset volatility on daily equity returns. Second, we assume that the book value of debt adequately captures the outstanding liabilities of the firm. For example, we do not consider non-debt liabilities in our baseline specification. Still, the Structural GARCH model is, at worst, effective in at least partially unlevering the firm.
} 
0.0676. For our subsample of firms, financial leverage accounts for roughly 17 percent of the leverage effect.

To put a bit more structure on the implications of Structural GARCH and the leverage effect, we run the following cross-sectional regression:

$$
\gamma_{E, i}-\gamma_{A, i}=a+b \times \overline{D / E}_{i}+\text { error }_{i}
$$

where $\gamma_{E, i}$ and $\gamma_{A, i}$ are the estimated GJR asymmetry parameter for firm $i$ 's equity returns and firm $i$ 's asset returns respectively. ${\bar{D} / E_{i}}_{i}$ is the mean debt to equity ratio for firm $i$ over the sample period. The logic behind the regression in (12) is simple - to the extent that leverage contributes to equity volatility asymmetry, firms with higher leverage should experience a larger reduction in volatility asymmetry after unlevering the firm. Table 3 presents the results.

Table 3: Equity Asymmetry versus Asset Asymmetry

\begin{tabular}{|c|c|c|c|}
\hline Variable & Coefficient Value & $t$-stat & $R^{2}$ \\
\hline$b$ & 0.0016 & 3.18 & $13.04 \%$ \\
\hline
\end{tabular}

Notes: This table presents the cross-sectional regression described in Equation (12). We regress the difference between the asymmetric volatility coefficient for equity and assets $\left(\gamma_{E, i}-\gamma_{A, i}\right)$ on the mean of the debt to equity ratio for each firm in our sample.

As expected, firms with higher average leverage have a larger gap between their equity and asset asymmetry. As we saw before, there is still a substantial amount of asset volatility asymmetry (the median $\gamma$ parameter for assets is 0.0676), which is helps explain why the $R^{2}$ is not higher. At the asset level, firms with higher volatility asymmetry should have higher risk premiums. Thus, as a rough quantitative exercise, we run the following two-stage 
regression:

$$
\begin{aligned}
& \text { Stage 1: } r_{i, t}^{A}=c+\beta_{m k t, i}^{A} r_{m k t, t}^{E}+e_{i, t} \\
& \text { Stage 2: } \gamma_{A, i}=e+f \times \beta_{m k t, i}^{A}+\varepsilon_{i}
\end{aligned}
$$

where $r_{m k t}^{E}$ is the return on the equity market index. Stage 1 of the regression is designed to deliver a measure of firms's risk premium through its CAPM beta. ${ }^{26}$ The coefficient $f$ in the Stage 2 regression is the main variable of interest. A positive value corroborates the risk premium story for volatility asymmetry. The results of the two-stage regression are found in Table 4.

Table 4: Risk-Premium Effect on Asset Asymmetry

\begin{tabular}{|c|c|c|c|}
\hline Variable & Coefficient Value & $t$-stat & $R^{2}$ \\
\hline$f$ & 0.0190 & 1.35 & $2.62 \%$ \\
\hline
\end{tabular}

Notes: This table presents the two-stage regression results in Equation (13). The first stage regression estimates, for each firm's asset return series, the equity market beta. The second stage regresses a measure of asset volatility asymmetry, the GJR asset $\gamma$, on the regression coefficient from Stage 1.

Unsurprisingly, firms with higher market betas have higher asset volatility asymmetry. Though the results are weak, we view them as qualitative confirmation for how the Structural GARCH unlevers the firm. Part of the reason for the standard error of our estimate of $f$ is that we compute $\beta_{m k t, i}^{A}$ at the firm level, and it is well known that betas are more precisely measured at the portfolio level. In addition, Bekaert and Wu (2000) attribute a portion of firm-level volatility asymmetry to covariance asymmetry between the market and the firm's equity. Our cross-sectional investigation does not include this (or other) potential

\footnotetext{
${ }^{26}$ Note that since we are interested in the cross-sectional behavior of $\gamma_{A, i}$ we are not concerned with using the return on the equity market. If, for example, we used some proxy for a broad market asset market index, only the magnitude of the coefficient $f$ would change.
} 
explanations, and is outside the scope of this paper. We now turn to using the Structural GARCH model to measure systemic risk.

\section{6. $\quad$ Systemic Risk Measurement}

Given the unprecedented rise in leverage and equity volatility during the financial crisis of 2007-09, systemic risk measurement is a natural application of the Structural GARCH model. Consider the following thought experiment: Following a negative shock to equity value, the financial leverage of the firm mechanically rises. In a simple asymmetric GARCH model for equity, the rise in volatility following a negative equity return is invariant to the capital structure of the firm. However, in the Structural GARCH model, the leverage multiplier will be higher following a negative equity return. Thus, equity volatility will be more sensitive to even slight rises in asset volatility. In simulating the model, this mechanism would manifest itself if the firm experiences a sequence of negative asset shocks. Due to asset volatility asymmetry, a sequence of negative asset returns increases asset volatility. In turn, there may potentially be explosive equity volatility since the leverage multiplier will be large in this case. Casual observation of equity volatility and leverage during the crisis clearly supports such a sequence of events.

\subsection{Traditional SRISK}

In order to embed this appealing feature of the Structural GARCH model into systemic risk measurement, we adapt the SRISK metric of Brownlees and Engle (2012) and Acharya, Pedersen, Philippon, and Richardson (2012). The reader should refer to these studies for an in-depth discussion of SRISK, but we will provide a brief summary here. Qualitatively, 
SRISK is an estimate of the amount of capital that an institution would need in order to function normally in the event of another financial crisis. To compute SRISK, we first compute a firm's marginal expected shortfall (MES), which is the expected loss of a firm when the overall market declines a given amount over a given time horizon. ${ }^{27}$ In turn, MES requires us to simulate a bivariate process for the firm's equity return, denoted $r_{i, t}^{E}$, and the market's equity return, denoted $r_{m, t}^{E}$. The bivariate process we adopt is described as follows:

$$
\begin{aligned}
r_{m, t}^{E} & =\sqrt{h_{m, t}^{E}} \varepsilon_{m, t} \\
r_{i, t}^{E} & =\sqrt{h_{i, t}^{E}}\left(\rho_{i, t} \varepsilon_{M, t}+\sqrt{1-\rho_{i, t}^{2}} \xi_{i, t}\right) \\
& =L M_{i, t-1} \sqrt{h_{i, t}^{A}}\left(\rho_{i, t} \varepsilon_{M, t}+\sqrt{1-\rho_{i, t}^{2}} \xi_{i, t}\right) \\
\left(\varepsilon_{m, t}, \xi_{i, t}\right) & \sim F
\end{aligned}
$$

where the shocks $\left(\varepsilon_{m, t}, \xi_{i, t}\right)$ are independent and identically distributed over time and have zero mean, unit variance, and zero covariance. We do not assume the two shocks are independent, however, and allow them to have extreme tail dependence nonparametrically. ${ }^{28}$ The processes $h_{m, t}^{E}, h_{i, t}^{E}$ and $\rho_{i, t}$ represent the conditional variance of the market, the conditional variance of the firm, and the conditional correlation between the market and the firm, respectively. It is important to note that under the Structural GARCH model, we are really estimating correlations between shocks to the equity market index and shocks to firm asset returns. Generically, once the bivariate process in (14) is fully specified, we compute a six month MES (henceforth LRMES for "long-run" marginal expected shortfall) by simulating the joint processes for the firm and the market (with bootstrapped shocks) and conditioning

\footnotetext{
${ }^{27}$ Acharya, Pedersen, Philippon, and Richardson (2012) provide an economic justification for why marginal expected shortfall is the proper measure of systemic risk in the banking system.

${ }^{28}$ See Brownlees and Engle (2012) for complete details.
} 
on the event that the market declines by 40 percent. Incorporating the Structural GARCH model into LRMES is simple. As stated in Equation (14), we simply assume the volatility process for firm equity returns follows a Structural GARCH model. ${ }^{29}$ Finally, we assume that equity market volatility follows a familiar GJR $(1,1)$ process and that correlations follow a $\operatorname{DCC}(1,1)$ model.

Once we have an estimate for the LRMES of a firm on a given day, we compute its capital shortfall in a crisis as follows:

$$
C S_{i, t}=k \operatorname{Debt}_{i, t}-(1-k)\left(1-L R M E S_{i, t}\right) E_{i, t}
$$

where $D_{e b t_{i, t}}$ is the book value of debt outstanding on the firm, $E_{i, t}$ is the market value of equity, and $k$ is a prudential level of equity relative to assets. In our applications, we take $k=8$ percent and, as is conventional in risk metrics such as VaR, we use positive values of $L R M E S_{i, t}$ to represent declines in the firm's value. For example, if firm $i$ is expected to lose 60 percent of its equity in a crisis, its LRMES will be 60 percent. Thus, positive values of capital shortfall mean the firm will be short of capital in a crisis. Finally, we define the SRISK of a firm as:

$$
S R I S K_{i, t}=\max \left(C S_{i, t}, 0\right)
$$

The parameters governing the market volatility and firm-market correlation are estimated recursively and allowed to change daily. However, due to the computational burden of estimating the Structural GARCH recursively each day, we use the full sample to estimate the Structural GARCH parameters. In future versions of SRISK measurement with Structural

\footnotetext{
${ }^{29}$ Efficient simulation of a bivariate Structural GARCH process is, however, not trivial. The MATLAB ${ }^{\circledR}$ code for this purpose is available from the authors upon request. All parameters of the model are estimated using our full sample of data for each firm.
} 
GARCH, we hope to estimate all parameters of the bivariate process recursively. In the interest of brevity, we choose to focus on one firm: Bank of America.

To start, Figure 10 plots the LRMES for Bank of America under using both the Structural GARCH, as well as a vanilla GJR model of univariate returns.

Figure 10: LRMES for Bank of America

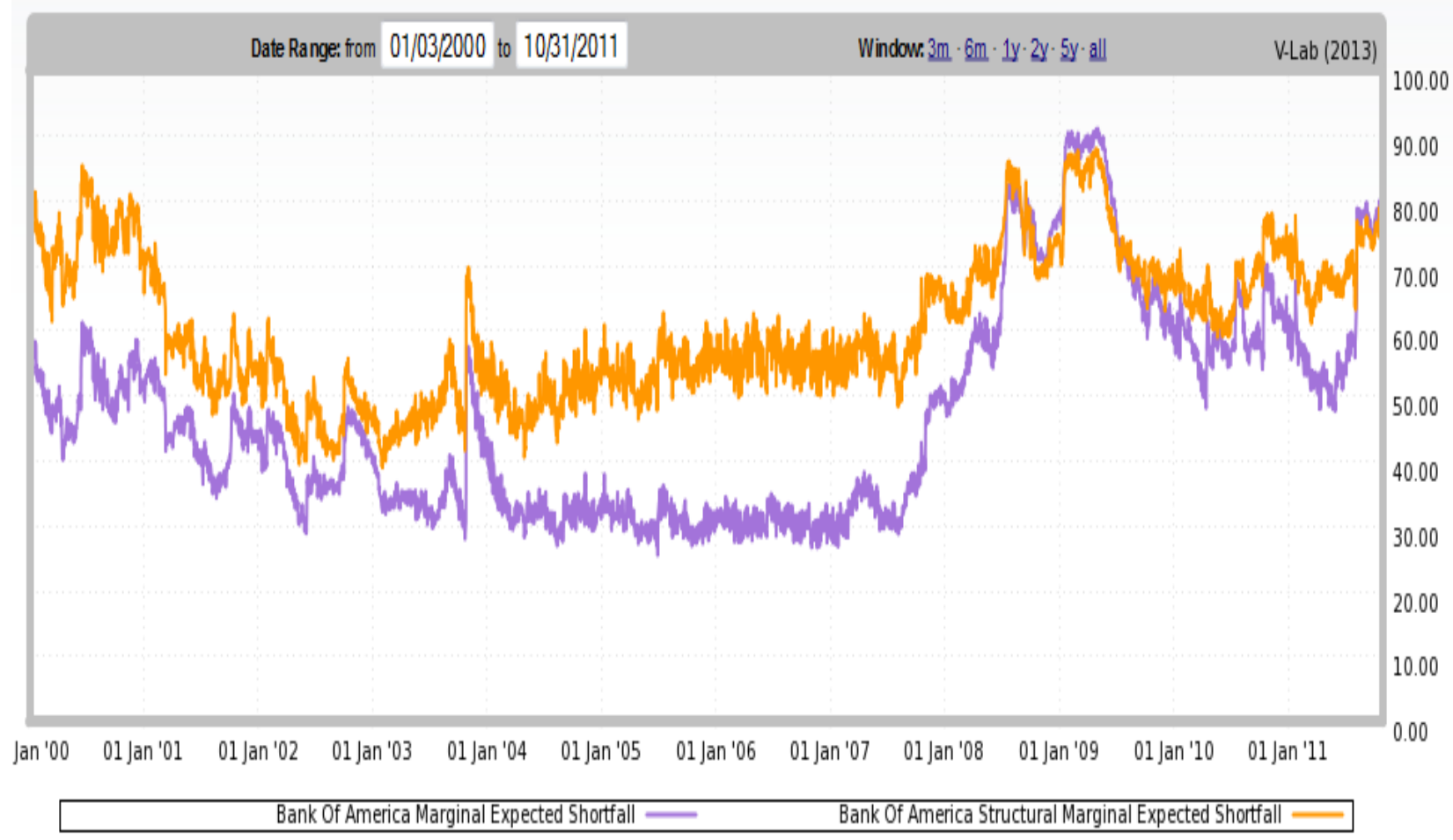

Notes: The figure above plots the Long Run Marginal Expected Shortfall (LRMES) of Bank of America. The purple line is the LRMES using a standard GJR model for returns. The orange line is the same calculation using the Structural GARCH model. The sample period is from January 2000 to November 2011.

It is obvious that the Structural GARCH induces a higher MES than a standard asymmetric volatility model. The reasons for this pattern are due to the leverage amplification mechanism built into the model directly. In the low-volatility period from 2004-2007, the Structural GARCH model delivers a LRMES nearly double the value that comes from a standard GJR. Even in this period of low leverage, there are negative equity paths in the 
Structural GARCH model that result in increases in leverage, which in turn result in higher volatility, and therefore paths where equity suffers large losses. There is much less of a scope for this type of leverage spiral in low volatility/leverage periods in our typical volatility models. To focus in on the recent financial crisis, we translate our LRMES calculations into SRISK and plot the resulting series from starting in 2007 in Figure 11.

Figure 11: SRISK for Bank of America

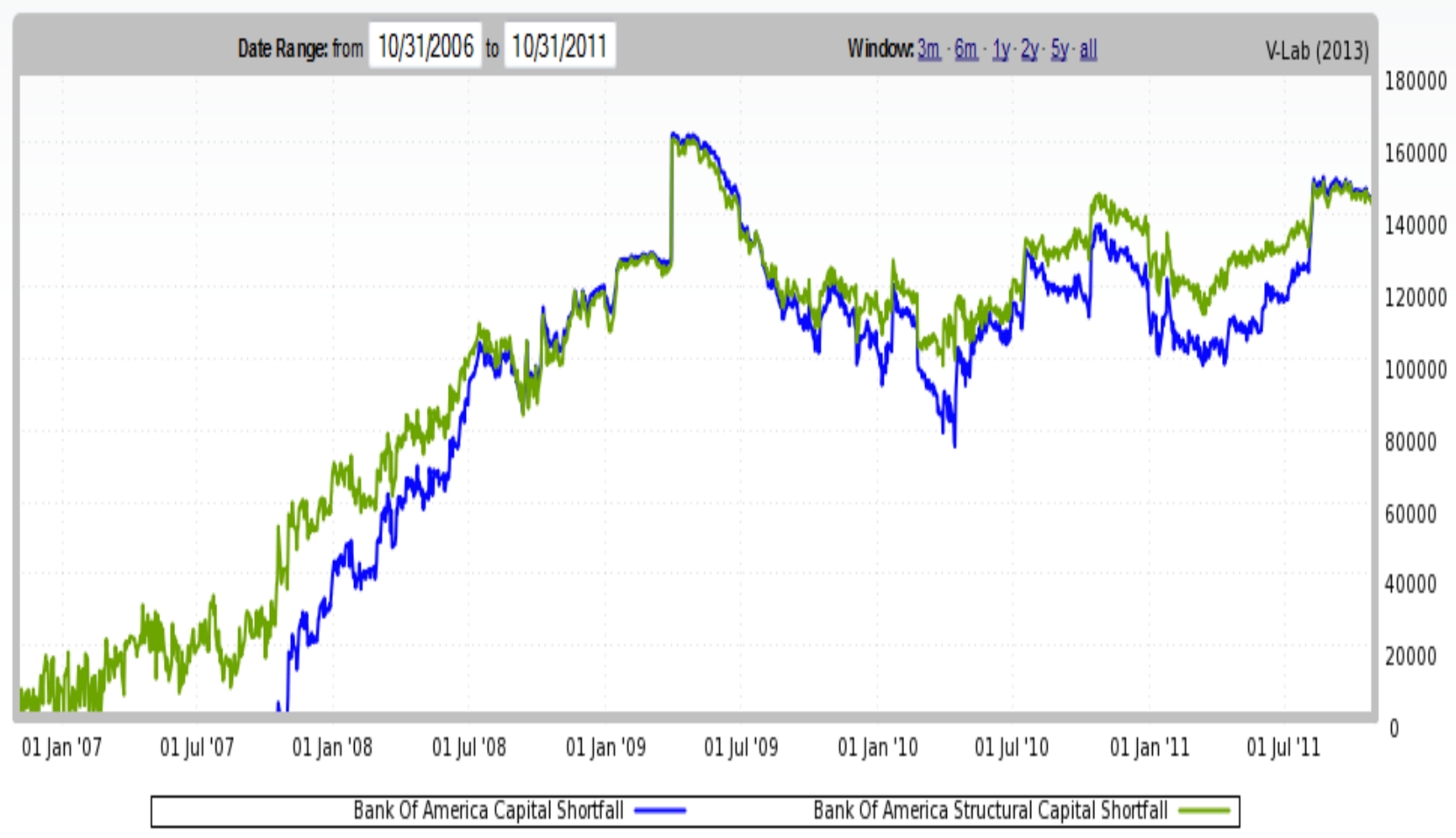

Notes: The figure above plots the SRISK of Bank of America from November 2006 to November 2011. The units of the $y$-axis are millions of USD. The blue line uses a standard GJR model for returns. The green line is the same calculation using the Structural GARCH model.

Figure 11 illustrates why the Structural GARCH model may provide useful in terms of providing early warning signals of threats to financial stability. The results echo the dynamics of LRMES under the Structural GARCH specification versus a standard GJR model. As 
early as January 2007, the SRISK (using Structural GARCH) of Bank of America starts to rise and hovers around $\$ 20$ billion. On the other hand, SRISK derived from a standard asymmetric volatility model shows no (expected) capital shortfalls until late 2007 and early 2008. Again, the success of the Structural GARCH along this dimension rests with the inherent leverage-volatility connection within the model. Qualitatively, the volatility and leverage link is apparent and has been discussed extensively in the media and the academic literature. Quantitatively, this link has been hard to pin down. The results in Figure 11 are evidence that the Structural GARCH model is, at very least, a partial resolution of this issue.

\subsection{Structural GARCH and the Probability of Default}

While the SRISK framework provides a way to assess the capital deficiencies of financial firms in a crisis, an alternative way to explore this issue is to ask the following: how likely is a firm to go bankrupt if crisis occurs? To answer this question, we use the same simulation machinery as in our SRISK analysis. Namely, for a given date, we condition on the market falling 40 percent over six months, and then we simulate future return paths of the firm using the bivariate specification in (14). We then compute the (conditional) probability of a firm going bankrupt as the proportion of simulated paths where bankruptcy occurs (i.e. when equity valuation hits zero). Our simulations again use two competing volatility models for returns: (i) the Structural GARCH and (ii) a standard GJR volatility model. For the purpose of illustration, we conduct this analysis for Bank of America on August 29, 2008.

Table 5 contains statistics on Bank of America's bankruptcy paths over various horizons and under both models. The connection between volatility and leverage is clearly seen in this setting as well, as the likelihood of Bank of America going bankrupt is five times higher under 
Table 5: Conditional Probability of Default

\begin{tabular}{|c|c|c|}
\hline & Structural GARCH & Regular GJR \\
\hline Total \# of Paths & 453 & 453 \\
\hline \# of Bankruptcies & 45 & 9 \\
\hline Probability of Bankruptcy & $9.93 \%$ & $1.99 \%$ \\
\hline Avg. Time to Bankruptcy & 89.4 & 91.2 \\
\hline Min Time to Bankruptcy & 42 & 57 \\
\hline Max Time to Bankruptcy & 126 & 126 \\
\hline
\end{tabular}

Notes: This table contains basic summary statistics for Bank of America's simulated bankruptcy paths starting from August 29, 2008. The simulations are: (i) over a six month horizon; (ii) conducted using the Structural GARCH and a GJR model; (iii) and are conditional on a drop of 40 percent in the aggregate market over the same timeframe. The number of simulations is 1841 .

the Structural GARCH model relative to the GJR model. At the end of August 2008, the Structural GARCH model indicated that Bank of America had a nearly 10 percent chance of going bankrupt should a crisis occur over the next six months, whereas the GJR model suggests only a 2 percent chance of this outcome. This is because the GJR model cannot hope to capture how leverage increases the future risk of the firm and makes bankruptcy more likely as the firm's leverage explodes. In addition, the minimum time to bankruptcy and the average time to bankruptcy are shorter in the Structural GARCH model, which is another way of depicting the type of volatility-leverage spirals our model is designed to encompass.

\subsection{A New Measure of Systemic Risk: Precautionary Capital}

Based on our preceding analysis of SRISK, it is clear that accounting for the interaction between volatility and leverage is extremely important. Our model provides a simple way to capture the nonlinear way in which increasing leverage increases current and future risk to equity holders, and vice versa. SRISK in one sense asks how much trouble would a financial 
firm be in the future if there is another crisis? However, within our framework we can ask a related, yet slight different, question: how much capital would the firm have to raise today to ensure they survive another crisis? In other words, our model provides a quantitative way to explore potential government policies that trade off between a firm holding precautionary capital buffers (crisis prevention) and the amount the firm would need should a crisis occur (bailouts). In our last application of the Structural GARCH model, we propose a new measure of systemic risk that we call "precautionary capital."

Let's start with a simple framework for understanding the core issues. Our ultimate goal is to think about the difference between today's equity, and how much equity the firm should have today to avoid a crisis in the future. Denote today's actual equity as $\widetilde{E}_{0}$, and book value of debt is $D_{0}$. Suppose further that, because of an equity injection, it is possible to change today's equity value from $\widetilde{E}_{0}$ to a different equity level of $E_{0}$.

Given initial levels of debt and equity, we then define the likelihood that future equity value, $E_{T}$, falls above any positive value $x$, conditional on a crisis:

$$
f\left(x ; E_{0}, D_{0}\right):=\mathbb{P}\left(E_{T} \geq x \mid \text { crisis }\right)
$$

In general, we allow the function $f(\cdot)$ to depend on the initial value of leverage, i.e. on $E_{0}$ and $D_{0}$. As with SRISK, our conditioning event of a crisis is a 40 percent drop in the aggregate stock market over the next six months. 
If we want the equity to asset ratio to be fixed in a crisis, it must be that:

$$
\begin{aligned}
k & =\frac{E_{T}}{E_{T}+D_{0}} \\
& \Leftrightarrow \\
E_{T} & =\frac{k D_{0}}{1-k}
\end{aligned}
$$

For ease of presentation, define the value of future of equity that meets the capital requirement as $\bar{E}_{T}\left(k, D_{0}\right):=\left(k D_{0}\right) /(1-k)$. Unless necessary, we drop the functional dependence of $\bar{E}_{T}$ on $k$ and $D_{0}$. Next, suppose we want to have some level of confidence, $\alpha \in[0,1]$, that the firm meets a capital requirement of $k$ in a crisis. Using the function $f(\cdot)$, we define the value $E_{0}^{*}$ such that:

$$
f\left(\bar{E}_{T} ; E_{0}^{*}, D_{0}\right)=\alpha
$$

Finally, we define precautionary capital as the difference between $E_{0}^{*}$ and the true value of today's equity:

$$
P C\left(k, \alpha ; \widetilde{E}_{0}, D_{0}\right):=E_{0}^{*}-\widetilde{E}_{0}
$$

In other words, precautionary capital measures how much additional capital would need to be added (or subtracted) to the equity of the firm to ensure, with a level of confidence of $\alpha$, that it meets its capital requirement in a crisis.

Computing $E_{0}^{*}$ and $P C\left(k, \alpha ; D_{0}\right)$ involves solving a complicated nonlinear root problem. It requires us to first compute the quantile function of $E_{T}$ as a function of $E_{0}$ and $D_{0}$, then to invert this function to solve for $E_{0}^{*}$. The problem is slightly easier when we are in the GARCH class of models. In this case, the quantile function will be invariant to the initial leverage of the firm. In the case of the Structural GARCH, the situation is more complicated, 
since the future return distribution depends crucially on today's leverage.

The central idea behind using Structural GARCH in measuring precautionary capital is precisely that adding equity to the firm today alters the quantiles of the future return distribution. Because the leverage multiplier is increasing in leverage, reducing leverage increases the likelihood the firm will meet its capital requirement. The effect is further enhanced by the fact that the leverage multiplier is concave in leverage. On the other hand, in a model without the volatility-leverage connection, reducing current leverage will not reduce future risk. ${ }^{30}$

Choosing $\mathbf{k}$ In order to compute precautionary capital, we must also select a value of $k$ that we'd like to ensure the firm meets in the event of a crisis. We choose a capital requirement of $k=2$ percent, which roughly translates to a leverage ratio of 50 . The reason for this particular capital requirement is simple: a leverage ratio of 50 seems to correspond to when a financial firm ceases its normal function (e.g. Lehman Brothers). Broadly speaking, the real effects of bank failures seem to take effect when financial firms stop providing their basic services; thus, $k=2$ percent can be thought of as a bankruptcy condition. Obviously, many different target capital ratios are also defensible.

\subsubsection{Example: Bank of America}

To make our idea more clear, we compute precautionary capital for Bank of America. For this particular exercise, we assume we are standing on October 1, 2008. In other words, we use initial values of $D_{0}$ and $\widetilde{E}_{0}$ as if we are at this point in time. Early October 2008 is of natural interest because it was just before the peak of the financial crises.

\footnotetext{
${ }^{30}$ Mathematically, this means that in the Structural GARCH, the function $f(\cdot)$ depends on leverage. In the GARCH family of volatility models, $f(\cdot)$ does not depend on leverage.
} 
Figure 12: Precautionary Capital: Bank of America on 10/1/2008

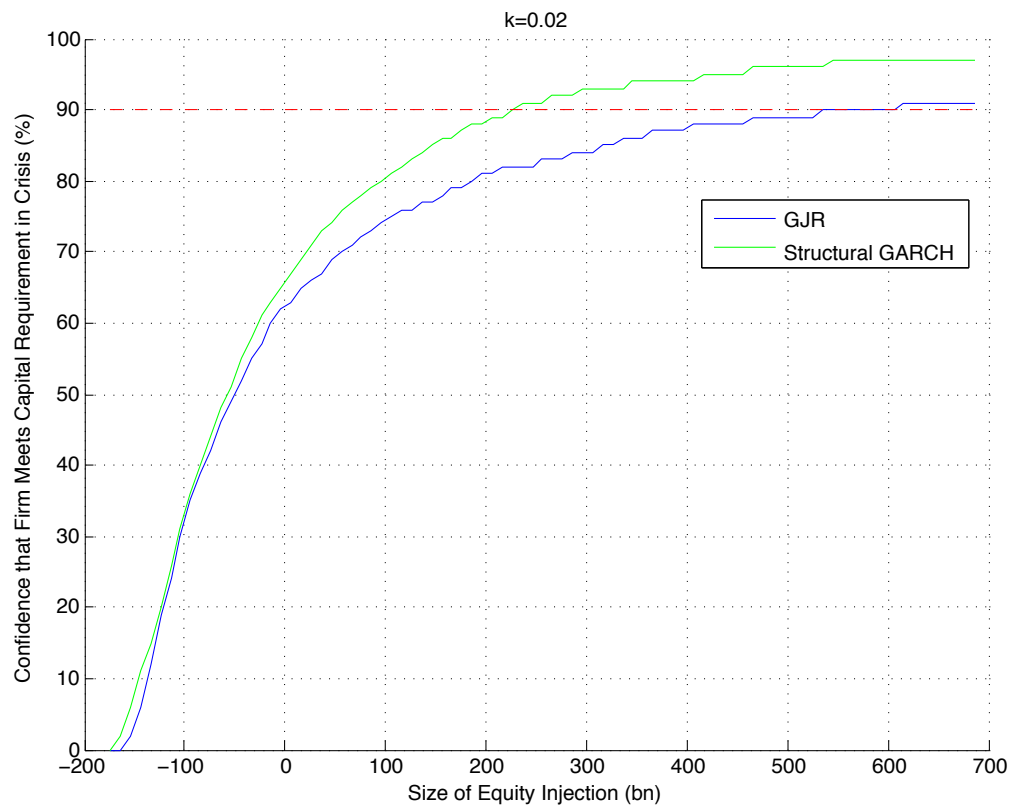

Notes: The figure above plots, for Bank of America, the likelihood of meeting a capital requirement against precautionary capital. The simulation date is on October 1, 2008. The debt level of the firm is $\$ 1.6701$ trillion. The true value of equity for the firm on this date is $\$ 173.87$ billion. The simulation horizon is six months and the number of simulations is 1841. Each set of simulations is conditioned on a drop of 40 percent in the aggregate stock market, and the same set of market and firm shocks is used for each simulation set the only difference is starting leverage. The curve in blue (green) is generated using a standard asymmetric GARCH model (Structural GARCH).

In Figure 12, we compute precautionary capital for different confidence levels. The $x$-axis in the plot is precautionary capital, or the size of a potential capital injection. The $y$-axis describes how likely the firm is to meet a $k=2$ percent capital requirement, for each level of precautionary capital. The curve in blue depicts our calculation of precautionary capital using a standard asymmetric GARCH model. The curve in green is the same computation, but uses the Structural GARCH model.

Figure 12 is useful because it allows a regulator to first choose a level of confidence for Bank of America to meet its capital requirement. The regulator can then say how much 
capital Bank of America would have needed on October 1, 2008 to be sure it meets this capital requirement if a crisis should occur in the next six months. For instance, according to a standard asymmetric GARCH model, in order to be 90 percent sure that Bank of America would meet a 2 percent capital requirement, we would have needed to inject nearly $\$ 600$ billion of equity in October 2008. This number is large because normal GARCH models ignore the volatility-leverage connection. According to the Structural GARCH model, we would have needed to add about $\$ 220$ billion to be 90 percent certain that Bank of America would meet its capital requirement.

With Structural GARCH, the leverage reduction of holding more capital results in lower volatility, lower beta and lower probability of failure, a very sensible outcome, but one which is not present in ordinary volatility models. In lieu of the advantages from reducing leverage, we believe these results imply that financial firms should be subject to a risk-based capital adequacy ratio for total leverage. Precautionary capital through the Structural GARCH lens provides a method to compute this type of capital adequacy ratio in a quantitatively precise manner.

\section{Conclusion}

This paper has provided a econometric approach to disentangle the effects of leverage on equity volatility. The Structural GARCH model we propose is rooted in the classical Merton (1974) structural model of credit, but departs from it in a flexible yet parsimonious way. In doing so, we are able to deliver high frequency asset return and asset volatility series. Our particular econometric parameterization is only one way in which to use the contingent claims analysis of Merton (1974) to capture the volatility-leverage connection; however, we 
believe the larger contribution of the current paper is to provide a more general framework through which to approach the problem.

Although we applied the model in two different settings, asymmetric volatility and systemic risk measurement, there are a variety of other settings in which our model can be used. For example, our analysis has centered on using structural models of credit to understand equity volatility dynamics; however, structural models of credit also make strong predictions for the volatility of credit spreads. Our framework may provide a better way to measure credit spread or credit default swap volatility by using the volatility-leverage connection highlighted in this paper, as well as observable equity shocks. Moreover, since the Structural GARCH model provides daily asset return and asset volatility series, it should also improve hedge ratios when looking at the sensitivity of a firm's debt value to the same firm's equity value. In corporate finance, an interesting avenue of research would be to examine optimal leverage ratios, taking into account the effect that the leverage multiplier has on equity volatility. In asset pricing, one might explore whether firms with high leverage multipliers are priced differently than firms with low leverage multipliers. The Structural GARCH model is an econometric model that can be used to explore all of these ideas. 


\section{References}

Viral Acharya, Lasse Pedersen, Thomas Philippon, and Matthew Richardson. Measuring systemic risk. NYU Stern, Working Paper, 2012.

Gurdip Bakshi, Charles Cao, and Zhiwu Chen. Empirical performance of alternative option pricing models. The Journal of Finance, 52(5):2003-2049, 1997. ISSN 1540-6261. doi: 10.1111/j.1540-6261.1997.tb02749.x. URL http://dx.doi.org/10.1111/j.1540-6261. 1997.tb02749.x.

Giovanni Barone-Adesi, Robert F. Engle, and Loriano Mancini. A garch option pricing model with filtered historical simulation. Review of Financial Studies, 21(3):1223-1258, 2008.

David Bates. Jumps and stochastic volatility: Exchange rate processes implicit in deutsche mark options. The Review of Financial Studies, 9(1):69-107, 1996.

G Bekaert and G Wu. Asymmetric volatility and risk in equity markets. Review of Financial Studies, 13(1):1-42, 2000.

Fischer Black. Studies of stock price volatility changes. Proceedings of the 1976 Meetings of the American Statistical Association, Business and Economics Statistics Section, pages 177-181, 1976.

Fischer Black and John C. Cox. Valuing corporate securities: Some effects of bond indenture provisions. The Journal of Finance, 31(2):351-367, 1976.

Fischer Black and Myron Scholes. The pricing of options and corporate liabilities. The journal of political economy, pages 637-654, 1973.

Tim Bollerslev. Generalized autoregressive conditional heteroskedasticity. Journal of Econometrics, 31(3):307-327, 1986.

Christian T Brownlees and Robert F Engle. Volatility, correlation and tails for systemic risk measurement. SSRN 1611229, 2012.

Jaewon Choi and Matt P. Richardson. The volatility of firm's assets and the leverage effect. SSRN: http://ssrn.com/abstract=1359368, 2012.

Andrew A Christie. The stochastic behavior of common stock variances: Value, leverage and interest rate effects. Journal of financial Economics, 10(4):407-432, 1982.

Pierre Collin-Dufresne and Robert S. Goldstein. Do credit spreads reflect stationary leverage ratios? The Journal of Finance, 56(5):1929-1957, 2001. 
Robert F Engle. Autoregressive conditional heteroscedasticity with estimates of the variance of united kingdom inflation. Econometrica, pages 987-1007, 1982.

Kenneth R French, G William Schwert, and Robert F Stambaugh. Expected stock returns and volatility. Journal of financial Economics, 19(1):3-29, 1987.

Lawrence R Glosten, Ravi Jagannathan, and David E Runkle. On the relation between the expected value and the volatility of the nominal excess return on stocks. The journal of finance, 48(5):1779-1801, 1993.

Jasmina Hasanhodzic and Andrew Lo. Black's leverage effect is not due to leverage. SSRN: http://ssrn.com/abstract=1762363, 2013.

Steven L Heston. A closed-form solution for options with stochastic volatility with applications to bond and currency options. Review of financial studies, 6(2):327-343, 1993.

Hayne E Leland and Klaus Bjerre Toft. Optimal capital structure, endogenous bankruptcy, and the term structure of credit spreads. The Journal of Finance, 51(3):987-1019, 1996.

Tim McQuade. Stochastic volatility and asset pricing puzzles. Stanford University GSB, Working Paper, 2013.

Robert C Merton. Theory of rational option pricing. The Bell Journal of economics and management science, pages 141-183, 1973.

Robert C Merton. On the pricing of corporate debt: The risk structure of interest rates*. The Journal of Finance, 29(2):449-470, 1974.

Robert C Merton. Option pricing when underlying stock returns are discontinuous. Journal of financial economics, 3(1):125-144, 1976.

Stephen M. Schaefer and Ilya A. Strebulaev. Structural models of credit risk are useful: Evidence from hedge ratios on corporate bonds. Journal of Financial Economics, 90(1):1 $-19,2008$.

Jeremy Stein and Elias Stein. Stock price distributions with stochastic volatility: An analytic approach. Review of Financial Studies, 4:727-752, 1991. URL http://rfs. oxfordjournals.org/. 


\section{A. Appendix: The Leverage Multiplier and Assumption 1 Under Different Option Pricing Models}

In this appendix, we do three things. First, we add jumps to the asset process. Second, we explore whether stochatic volatility and/or jumps change the conclusions drawn in the main text regarding using Equation (9) as a model of equity returns. Finally, we confirm that jumps and stochastic volatility do not change the general shape of the leverage multiplier, and so our specification remains flexible enough to be accurate in these settings. Through this exposition, the steps to construct Figure 3 will also become clear. In order to accomplish these tasks, it will be useful to essentially start from scratch, and assign a generalized process for assets. As such, the reader may find many parts repetitive from the main text, but we take this approach for the sake of completeness.

\section{A.1. Motivating the Model}

Adding jumps means we re-define the equity value as follows:

$$
E_{t}=f\left(A_{t}, D_{t}, \sigma_{A, t}, J_{A}, N_{A}, \tau, r_{t}\right)
$$

where $f(\cdot)$ is an unspecified call option function, $A_{t}$ is the current market value of assets, $D_{t}$ is the current book value of outstanding debt, $\sigma_{A, t}$ is the (potentially stochastic) volatility of the assets. $\tau$ is the life of the debt, and finally, $r_{t}$ is the annualized risk-free rate at time $t$. Additionally, $J_{A}$ and $N_{A}$ are processes that describe discontinuous jumps in the underlying assets. Next, we specify the following generic process for assets and variance:

$$
\begin{aligned}
\frac{d A_{t}}{A_{t}} & =\left[\mu_{A}(t)-\lambda \mu_{J}\right] d t+\sigma_{A, t} d B_{A}(t)+J_{A} d N_{A}(t) \\
d \sigma_{A, t}^{2} & =\mu_{v}\left(t, \sigma_{A, t}\right) d t+\sigma_{v}\left(t, \sigma_{A, t}\right) d B_{v}(t)
\end{aligned}
$$

where $d B_{A}(t)$ is a standard Brownian motion. $\sigma_{A, t}$ captures time-varying asset volatility. We also capture potential jumps in asset values via $J_{a}$ and $N_{A}(t) . \log \left(1+J_{A}\right) \sim N\left(\log \left[1+\mu_{J}\right]-\sigma_{J}^{2} / 2, \sigma_{J}^{2}\right)$

and $N_{t}$ is a Poisson counting process with intensity $\lambda$. The relative price jump size $J_{A}$ determines the percentage change in the asset price caused by jumps, and the average asset jump size is $\mu_{J}$. We assume the jump size, $J_{A}$, is independent of $N_{A}(t), B_{A}(t)$, and $B_{v}(t)$. Similarly, the asset Poisson counting process $N_{A}(t)$ is assumed to be independent of $B_{A}(t)$ and $B_{v}(t)$. We allow an arbitrary instantaneous correlation of $\rho_{t}$ between the shock to asset returns, $d B_{A}(t)$, and the shock to asset volatility, $d B_{v}(t)$.

The instantaneous return on equity is computed via simple application of Itō's Lemma for 
Poisson processes:

$$
\begin{aligned}
\frac{d E_{t}}{E_{t}}= & \Delta_{t} \frac{A_{t}}{D_{t}} \frac{D_{t}}{E_{t}} \cdot \frac{d A_{t}}{A_{t}}+\frac{\nu_{t}}{E_{t}} \cdot d \sigma_{A, t}+\frac{1}{2 E_{t}}\left[\frac{\partial^{2} f}{\partial A_{t}^{2}} d\langle A\rangle_{t}+\frac{\partial^{2} f}{d\left(\sigma_{A, t}\right)^{2}} d\left\langle\sigma_{A}^{f}\right\rangle_{t}+\frac{\partial^{2} f}{\partial A \partial \sigma_{A, t}} d\left\langle A, \sigma_{A}\right\rangle_{t}\right] \\
& +\left[\frac{E_{t}^{J}-E_{t}}{E_{t}}\right] d N_{A}(t)
\end{aligned}
$$

where $\Delta_{t}=\partial f\left(A_{t}, D_{t}, \sigma_{A, t}, \tau, r\right) / \partial A_{t}$ is the "delta" in option pricing, $\nu_{t}=\partial f\left(A_{t}, D_{t}, \sigma_{A, t}, \tau, r\right) / \partial \sigma_{A, t}$ is the so-called "vega" of the option, and $\langle X\rangle_{t}$ denotes the quadratic variation process for an arbitrary stochastic process $X_{t}$. Additionally, $E_{t}^{J}$ is the value of equity for an asset jump of $J_{A}=J$. Hence, $E_{t}^{J}$ is itself a random variable. Once again, we have ignored the sensitivity of the option value to the maturity of the debt. All the quadratic variation terms are of the order $\mathcal{O}(d t)$ and henceforth we collapse them to an unspecified function $q\left(A_{t}, \sigma_{A, t} ; f\right)$, where the notation captures the dependence of the higher order Itō terms on the partial derivatives of the call option pricing function.

The call option pricing function is still monotonically increasing in its first argument, so it is safe to assume that $f(\cdot)$ is invertible with respect to this argument. We further assume that the call pricing function is homogenous of degree one in its first two arguments. Define the inverse call option formula as follows:

$$
\begin{aligned}
\frac{A_{t}}{D_{t}} & =g\left(E_{t} / D_{t}, 1, \sigma_{A, t}, J_{A}, N_{A}, \tau, r_{t}\right) \\
& \equiv f^{-1}\left(E_{t} / D_{t}, 1, \sigma_{A, t}, J_{A}, N_{A}, \tau, r_{t}\right)
\end{aligned}
$$

Thus, Equation (21) reduces returns to the following: ${ }^{31}$

$$
\begin{aligned}
\frac{d E_{t}}{E_{t}} & =\overbrace{\Delta_{t} \cdot g\left(E_{t} / D_{t}, 1, \sigma_{A, t}^{f}, \tau, r_{t}\right) \cdot \frac{D_{t}}{E_{t}}}^{\equiv L M\left(E_{t} / D_{t}, 1, \sigma_{A, t}, \tau, r_{t}\right)} \frac{d A_{t}}{A_{t}}+\frac{\nu_{t}}{E_{t}} \cdot d \sigma_{A, t}+q\left(A_{t}, \sigma_{A, t}^{f} ; f\right) d t+\left[\frac{E_{t}^{J}-E_{t}}{E_{t}}\right] d N_{A}(t) \\
& =\operatorname{LM}\left(E_{t} / D_{t}, 1, \sigma_{A, t}, \tau, r_{t}\right) \times \frac{d A_{t}}{A_{t}}+\frac{\nu_{t}}{E_{t}} \cdot d \sigma_{A, t}+q\left(A_{t}, \sigma_{A, t}^{f} ; f\right) d t+\left[\frac{E_{t}^{J}-E_{t}}{E_{t}}\right] d N_{A}(t) \quad(23)
\end{aligned}
$$

Henceforth, when it is obvious, we will drop the functional dependence of the leverage multiplier on leverage, etc. and instead denote it simply by $L M_{t}$. In order to obtain a complete law of motion

\footnotetext{
${ }^{31}$ Using the fact that $f(\cdot)$ is homogenous of degree 1 in its first argument also implies that

$$
\Delta_{t}=\partial f\left(A_{t}, D_{t}, \sigma_{A, t}, \tau, r\right) / \partial A_{t}=\partial f\left(A_{t} / D_{t}, 1, \sigma_{A, t}, \tau, r\right) / \partial\left(A_{t} / D_{t}\right)
$$

So with an inverse option pricing formula, $g(\cdot)$ in hand we can define the delta in terms of leverage $E_{t} / D_{t}$.
} 
for equity, we use Itō's Lemma to derive the volatility process:

$$
\begin{aligned}
d \sigma_{A, t} & =\overbrace{\left[\frac{\mu_{v}\left(t, v_{t}\right)}{2 \sigma_{A, t}}-\frac{\sigma_{v}^{2}\left(t, v_{t}\right)}{8 \sigma_{A, t}^{3}}\right]}^{\equiv s\left(\sigma_{A, t} ; \mu_{v}, \sigma_{v}\right)} d t+\frac{\sigma_{v}\left(t, v_{t}\right)}{2 \sigma_{A, t}} d B_{v}(t) \\
& =s\left(\sigma_{A, t} ; \mu_{v}, \sigma_{v}\right) d t+\frac{\sigma_{v}\left(t, v_{t}\right)}{2 \sigma_{A, t}} d B_{v}(t)
\end{aligned}
$$

Plugging Equations (20), (24) into Equation (23) yields the desired full equation of motion for equity returns:

$$
\begin{aligned}
\frac{d E_{t}}{E_{t}}= & {\left[L M_{t} \mu_{A}(t)+s\left(\sigma_{A, t} ; \mu_{v}, \sigma_{v}\right)+q\left(A_{t}, \sigma_{A, t} ; f\right)\right] d t } \\
& +L M_{t} \sigma_{A, t} d B_{A}(t)+\frac{\nu_{t}}{E_{t}} \frac{\sigma_{v}\left(t, \sigma_{A, t}\right)}{2 \sigma_{A, t}} d B_{v}(t)+\left[\frac{E_{t}^{J}-E_{t}}{E_{t}}\right] d N_{A}(t)
\end{aligned}
$$

Since typical daily equity returns are virtually zero on average, we can continue to ignore the equity drift term. Instantaneous equity returns then naturally derive from Equation (25) with no drift:

$$
\frac{d E_{t}}{E_{t}}=L M_{t} \sigma_{A, t} d B_{A}(t)+\frac{\nu_{t}}{E_{t}} \frac{\sigma_{v}\left(t, \sigma_{A, t}\right)}{2 \sigma_{A, t}} d B_{v}(t)+\left[\frac{E_{t}^{J}-E_{t}}{E_{t}}\right] d N_{A}(t)
$$

Our ultimate object of interest is the instantaneous volatility of equity, but in order to obtain a complete expression for equity volatility we have to determine the variance of the jump component of equity returns. In Appendix B, we derive an easily computed expression, denoted by $\mathcal{V}_{A}^{J}\left(J_{A}, N_{A}(t) ; A_{t}\right)$, that involves a simple integration over the normal density. Hence, total instantaneous equity volatility in this (reasonably) general setting is given by:

$$
\operatorname{vol}_{t}\left(\frac{d E_{t}}{E_{t}}\right)=\sqrt{L M_{t}^{2} \times \sigma_{A, t}^{2}+\frac{\nu_{t}^{2} \sigma_{v}^{2}\left(t, \sigma_{A . t}\right)}{4 E_{t}^{2} \sigma_{A, t}^{2}}+2 L M_{t} \sigma_{A, t} \frac{\nu_{t}}{E_{t}} \frac{\sigma_{v}\left(t, \sigma_{A, t}\right)}{2 \sigma_{A, t}} \rho_{t}+\mathcal{V}_{A}^{J}\left(J_{A}, N_{A}(t) ; A_{t}\right)}
$$

There are four terms that contribute to equity volatility. The first term relates to asset volatility and the second relates to the volatility of asset volatility (as well as the sensitivity of the option to changes in volatility). The third depends on the correlation between assets innovations and asset volatility innovations. In practice, this correlation is negative. Thus, the middle two terms in Equation (27) will have offsetting effects in terms of the contribution of stochastic asset volatility to equity volatility. Finally, the fourth term relates to the volatility of the jump process for assets. In later sections and also in the Appendix C, we show that we can ignore all but the first term for the purposes of volatility modeling in our context because, compared to asset volatility, they contribute very little to equity volatility. Thus, Equation (26) and (27) reduce to a very simple expression for 
equity returns and instantaneous volatility, and is the basis for Assumption 1:

$$
\begin{aligned}
\frac{d E_{t}}{E_{t}} & \approx L M_{t} \sigma_{A, t} d B_{A}(t) \\
\operatorname{vol}_{t}\left(\frac{d E_{t}}{E_{t}}\right) & \approx L M_{t} \times \sigma_{A, t}
\end{aligned}
$$

Equation (28) is our key relationship of interest. It states that equity volatility (returns) is a scaled function of asset volatility (returns), where the function depends on financial leverage, $D_{t} / E_{t}$, as well as asset volatility over the life of the option (and the interest rate). The moniker of the "leverage multiplier" should be clear now. The functional form for $L M(\cdot)$ depends on a particular option pricing model, and one of our key contributions is to estimate a generalized $L M(\cdot)$ function that, in practice, encompasses a number of option pricing models. In order to build further intuition for the properties of the leverage multiplier, we examine it in three different option pricing models: Merton (1976), Heston (1993), and Bates (1996).

\section{A.2. The Leverage Multiplier Under Various Option Pricing Mod- els}

The purpose of this subsection is twofold. We first aim to show that the approximation in Equation (28) performs well in a variety of different option pricing models. The main mechanism behind this approximation is as follows: as the time to maturity of the equity option gets larger, the contribution of the volatility of volatility (and jumps, if small enough in probability) to equity returns/volatility is minimal. This is primarily because volatility is mean reverting, and thus "long run" volatility is effectively constant, so that short run changes in volatility matter little for equity returns. In all of the option pricing models we consider, we assess the accuracy of this approximation in the same way. We simply parameterize the model and compute the total volatility of equity, given by Equation (27), within the model. Then, we compare it with the approximation of (28) within the same model. This comparison is conducted at debt maturities ranging from one month to twenty years. For each maturity, we choose the debt level such that the leverage is the same across maturities. For example, suppose we are examining Option Model A. For each maturity, we search for the debt (strike) such that debt to equity will be some arbitrary level $L$. For this debt level, we next compute the total equity volatility and the approximation under Option Model A; then we repeat this process for each maturity. We opt to keep leverage consistent across maturities since our empirical work will focus on financial firms whose leverage tends to be high, despite heterogenous debt maturities. The leverage level we target is $L=15$. Why this number for leverage? For the empirical portion of our investigation, we examine financial firms. Financial firms typically have high levels of leverage ranging from 10-20, where leverage is measured as the book value of debt divided by the market value of equity. When possible, we are also careful to keep parameters consistent across all option pricing models we consider in order to keep the results comparable.

After we establish the validity of the approximation in Equation (28), we explore how the leverage multiplier behaves in a variety of option pricing settings. As we will show, Equation (28) 
is indeed a useful approximation, and the leverage multiplier takes on a similar shape across many different models.

\section{A.2.1. The Heston (1993) Model}

In order to justify Assumption 1, we study equity dynamics under the popular Heston (1993) stochastic volatility model. In this setting, asset volatility evolves as follows:

$$
d \sigma_{A, t}^{2}=\kappa\left[\theta-\sigma_{A, t}^{2}\right] d t+\eta \sigma_{A, t} d B_{v}(t)
$$

The parameter $\kappa$ determines how quickly volatility mean reverts, $\theta$ determines the asymptotic limit of volatility and $\eta$ tunes the volatility of volatility. ${ }^{32}$ This specification of asset returns can be viewed as a special case of Equation (2).

Validating Assumption 1 Our first task is to analyze how equity volatility is affected by this particular stochastic volatility model. In this model, it is straightforward from Equation (8) that the total volatility of equity is given by:

$$
\operatorname{vol}_{t}\left(\frac{d E_{t}}{E_{t}}\right)=\sqrt{L M_{t}^{2} \times \sigma_{A, t}^{2}+\frac{\nu_{t}^{2} \eta^{2}}{4 E_{t}^{2}}+L M_{t} \sigma_{A, t} \frac{\eta \nu_{t}}{E_{t}} \rho_{t}\left(d B_{A}(t), d B_{v}(t)\right)}
$$

Let us consider the magnitudes of each of the terms that contribute to equity volatility. The closed form option pricing formula in this setting was the major contribution of Heston (1993), and the greeks we are interested in do have closed forms, but we choose to compute them via numerical derivatives. For the remainder of this subsection, we assume that risk-neutral asset and volatility processes are specified by the Heston (1993) model, which we parametrize as summarized in Table 6.

Table 6: Heston (1993) Calibration for Risk-Neutral Asset Prices

\begin{tabular}{|c|c|}
\hline Parameter & Value \\
\hline$\kappa$ & 4 \\
\hline$\theta$ & $0.15^{2}$ \\
\hline$\eta$ & 0.15 \\
\hline$\sigma_{A, t}$ & 0.15 \\
\hline$\rho$ & -0.5 \\
\hline
\end{tabular}

Notes: This table provides the parameters we use to calibrate the risk-neutral asset return process under the Heston (1993) model.

\footnotetext{
${ }^{32}$ For a more technical description of the properties of this volatility process see Heston (1993) or Cox, Ingersoll, and Ross (1985).
} 
Setting $\kappa=4$ sets the half-life of the volatility process to be approximately 44 days, which is roughly the half-life of the GARCH processes we typically encounter in practice. ${ }^{33}$ Setting $\theta=0.15^{2}$ means that long-run asset volatility is $15 \%$ in annualized terms, which we adopt to keep consistency with the analysis in Section 2.2.1 (we also initialize current spot volatility to its long-run average). $\eta=0.15$ makes the volatility of asset volatility to be $15 \%$ annually, or equal to the volatility of assets returns themselves. To the best of our knowledge, this does not contradict known fact. ${ }^{34}$ Finally, we set the correlation between asset volatility shocks and asset return shocks to be -0.5 in order to capture the well-known asymmetry in volatility.

Our objective is to show that under the Heston (1993) specification, it is perfectly reasonable to ignore the terms in Equation (8) that are related to stochastic asset volatility. The argument for ignoring the stochastic portion of asset volatility in terms of its contribution to equity volatility hinges on the idea that for longer maturity debt, the volatility of asset volatility contributes very little to equity volatility. Thus, under the current parameterization of the Heston (1993) model, we plot the total instantaneous equity volatility against maturity in Equation (29) against maturity. In addition, we plot instantaneous equity volatility under Assumption 1. ${ }^{35}$ Figure 13 provides a visualization of the results.

As should be clear, the assumption underlying Equation (9) is quite reasonable in terms of capturing total equity volatility. The reason is due entirely to the mean-reversion property of asset volatility. Because asset volatility mean reverts quickly relative to the life of the debt, the "vega" of equity is very small. To see this point more forcefully, consider equity returns (with zero drift) if assets follows the Heston model:

$$
\frac{d E_{t}}{E_{t}}=L M_{t} \sigma_{A, t} d B_{A}(t)+\frac{\eta \nu_{t}}{2 E_{t}} d B_{v}(t)
$$

How big is each term multiplying the shocks in this model? Staying with the parameters in Table 6, we plot both in Figure 14.

Clearly, the contribution of volatility shocks to equity returns is minimal. This result shouldn't be surprising as it is essentially a restatement of the conclusion reached from Figure $13 .{ }^{36}$

\footnotetext{
${ }^{33}$ The half-life of the volatility process is $\ln (2) / \kappa$.

${ }^{34}$ In fact, this may even be too large. As assets are much less volatile than equity, so one might expect the volatility of asset volatility to be smaller than the volatility of equity volatility as well.

${ }^{35}$ For each maturity, we search for the debt (strike) such that debt to equity will be some arbitrary level $L$. For this debt level, we next compute the total equity volatility and the approximation under the Heston (1993) model; then we repeat this process for each maturity. We opt to keep leverage consistent across maturities since our empirical work will focus on financial firms whose leverage tends to be high, despite heterogenous debt maturities. The leverage level we target is $L=15$. Why this number for leverage? For the empirical portion of our investigation, we examine financial firms. Financial firms typically have high levels of leverage ranging from 10-20, where leverage is measured as the book value of debt divided by the market value of equity.

${ }^{36}$ Hence, we arrive at the previously assumed process for equity returns, even in a stochastic volatility environment:

$$
\frac{d E_{t}}{E_{t}}=L M_{t} \times \sigma_{A, t} d B_{A}(t)
$$
}


Figure 13: Equity Volatility Analysis in Heston (1993) Model

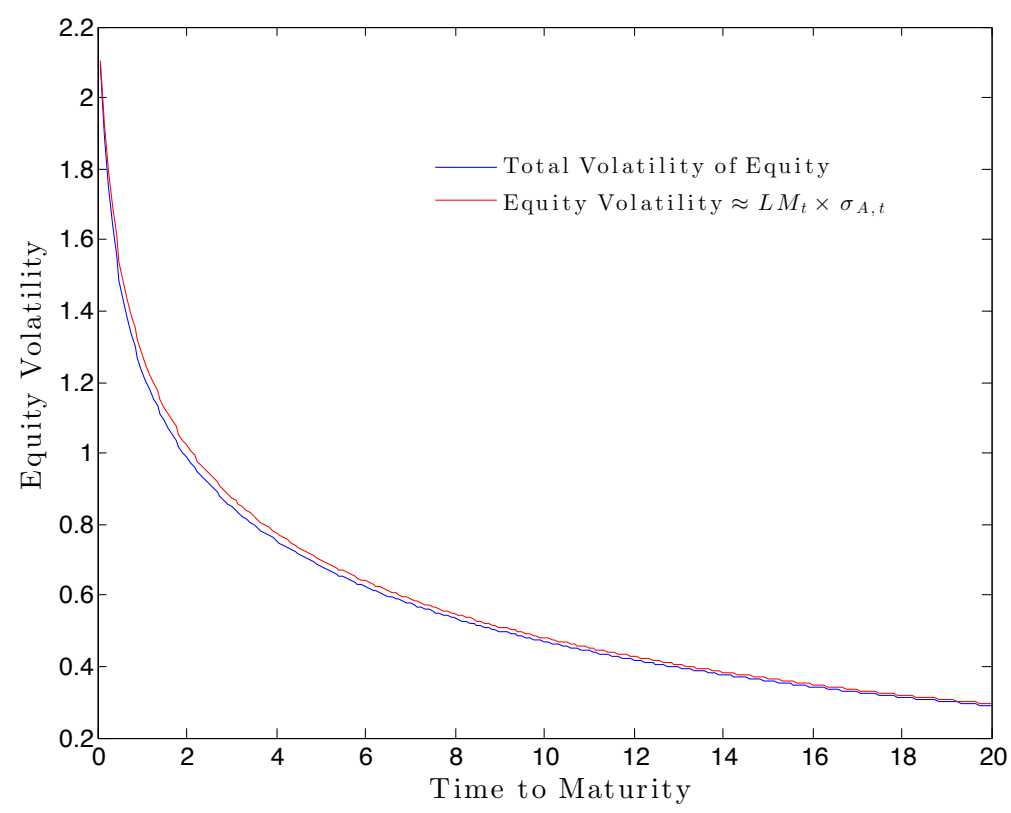

Notes: The blue line in this figure plots the total (true) volatility of equity if assets follow the Heston (1993) model. The red line in this figure plots the volatility of equity if it is approximated according to Equation (9). For each time to maturity, we set $A_{t}=1$ and choose the level of debt such that financial leverage is approximately 15. For this analysis, the risk-free rate is set to 0.03 .

The Leverage Multiplier in the Heston (1993) Model Now that we have established that the leverage multiplier is the key determinant of equity dynamics in this stochastic volatility environment, we turn to a more careful analysis of the actual shape of the leverage multiplier. Figure 15 plots the leverage multiplier (for debt of maturity $\tau=5$ ) against leverage as we did in the BSM case.

The shape of the leverage multiplier under stochastic volatility is quite similar to the BSM case. The most obvious difference is the concavity of the stochastic volatility leverage multiplier. When moving from low levels of leverage (e.g. 2-3) to intermediate levels of leverage (e.g. 5-7) the leverage multiplier rises rapidly as compared to the BSM baseline. In addition, the level of the stochastic volatility leverage multiplier is higher than the BSM counterpart. Because of the negative correlation between asset returns and asset volatility, the future asset return distribution has negative skewness. In turn, leverage has strong effects on equity returns and volatility since the option value of equity is less likely to expire in the money. This intuition is also consistent with the results of section 2.2.3, where we use Monte Carlo simulation to explore models with stochastic volatility and non-normal shocks.

This isn't to say that the volatility of volatility terms do not matter. They matter quite a bit for the level of equity, but not so much for the returns. 
Figure 14: Equity Return Analysis in Heston (1993) Model

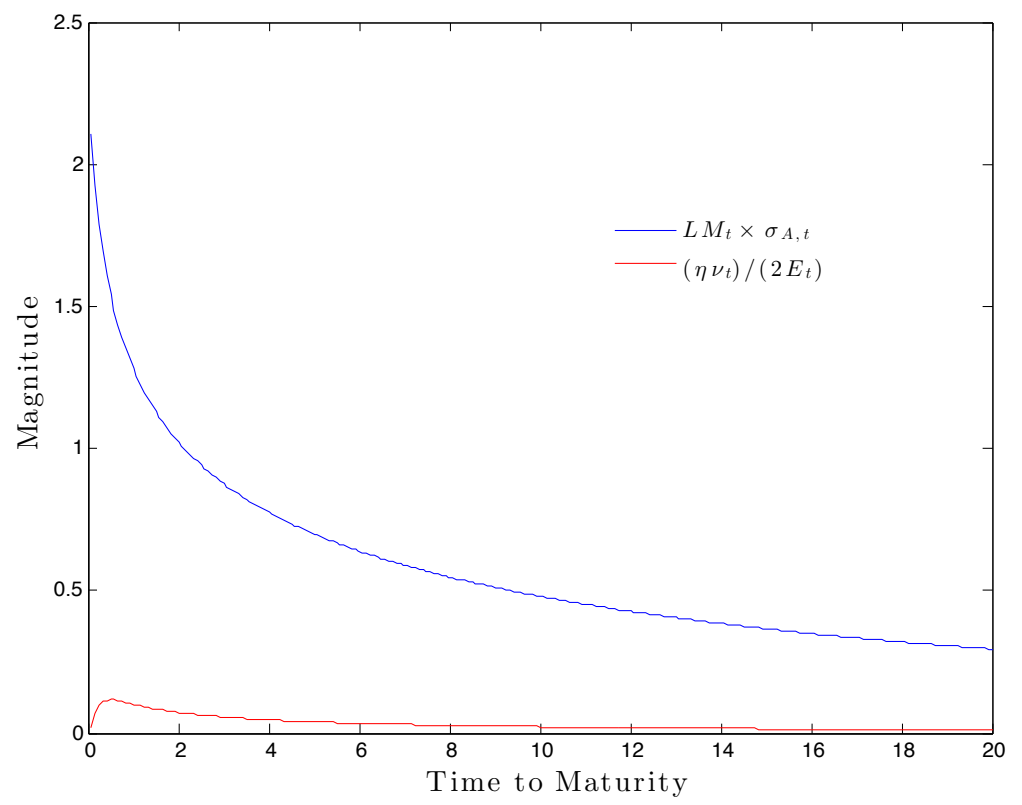

Notes: The blue line in this figure plots the how much asset return shocks are magnified into equity shocks. The red line in this figure plots how much volatility shocks are amplified into equity shocks. For each time to maturity, we set $A_{t}=1$ and choose the level of debt such that financial leverage is approximately 15. For this analysis, the risk-free rate is set to zero.

\section{A.2.2. Analysis of the Leverage Multiplier under Merton's (1976) Jump-Diffusion Model}

Another strand of option pricing models in the literature began with the seminal work of Merton (1976), henceforth MJD. In this formulation, the stock returns follow a standard geometric Brownian motion, appended with a continuous time Poisson jump process. Again, this case is encompassed by the specification in Equation (26), but we turn off the stochastic volatility channel (i.e. $\sigma_{A, t}=\sigma_{A}$ ) and allow only for jumps. In this setting, equity return dynamics from (26) reduce to the following jump-diffusion:

$$
\frac{d E_{t}}{E_{t}}=L M_{t} \sigma_{A} d B_{A}(t)+\left[\frac{E_{t}^{J}-E_{t}}{E_{t}}\right] d N_{A}(t)
$$

Similarly, the volatility of equity returns reduces to the following:

$$
\operatorname{vol}_{t}\left(\frac{d E_{t}}{E_{t}}\right)=\sqrt{L M_{t}^{2} \times \sigma_{A}^{2}+\mathcal{V}_{A}^{J}\left(J_{A}, N_{A}(t) ; A_{t}\right)}
$$

where again the expression for $\mathcal{V}_{A}^{J}\left(J_{A}, N_{A}(t) ; A_{t}\right)$ is found in Appendix B. The key insight of MJD model was that, even though dynamic riskless hedging is impossible with discontinuous sample paths, if individual stock jumps are independent of the prevailing pricing kernel then their presence 
Figure 15: Leverage Multiplier in Heston (1993) Model

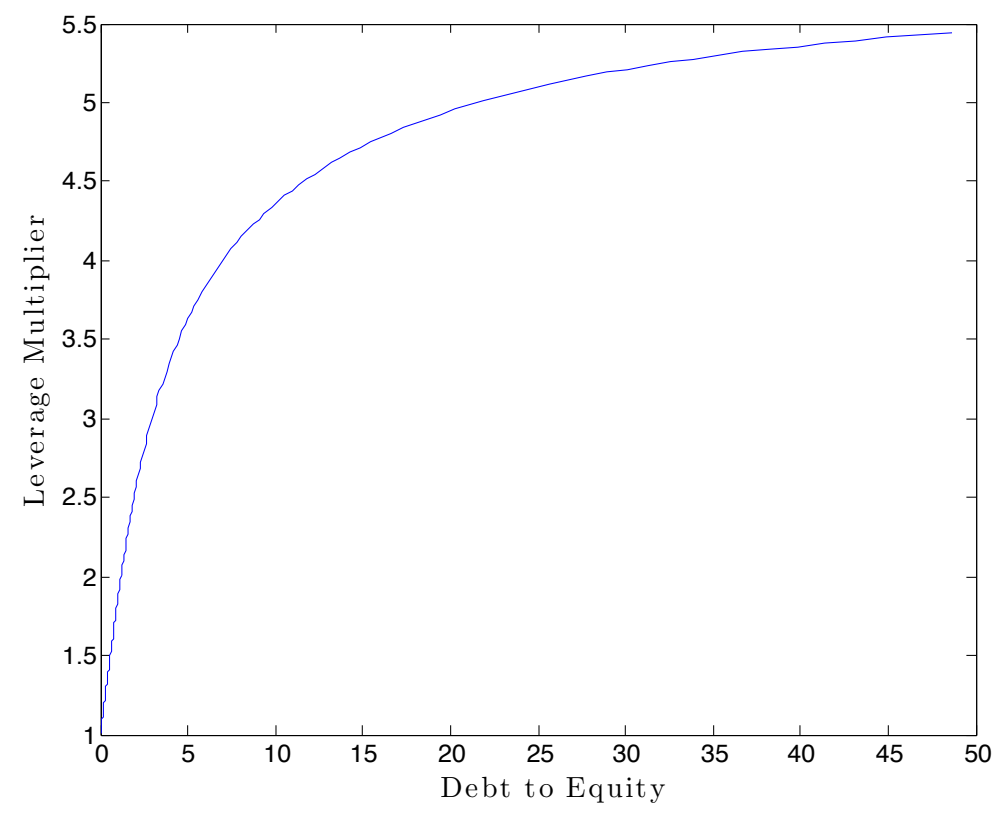

Notes: We plot the leverage multiplier in the Heston (1993) stochastic volatility model against leverage. The time to maturity is set to five years and the remaining model parameters are given in Table 6. For this analysis, the risk-free rate is set to $r=0.03$.

is an "unpriced" risk and typical hedging arguments can still be applied. Merton (1976) solves for the closed form option pricing solution, which turns out to be a weighted average of BSM prices, with the weights determined by the likelihood of a given number of jumps over the life of the debt. ${ }^{37}$ Our analysis of his solution begins with an assessment of the approximation in Equation (28).

Validating Assumption 1 In order to do so, we first parameterize the MJD model as in Table 7 .

\footnotetext{
${ }^{37}$ To use the exact Merton (1976) formula in our context, set the mean of the "Merton Jump", which is $\log$-normal to $\mu_{M}=\log \left(1+\mu_{J}\right)-\sigma_{J}^{2} / 2$ in the specification we outline for jumps. The variance is the same.
} 
Figure 16: Accuracy of Volatility Approximation in Merton (1976) Model

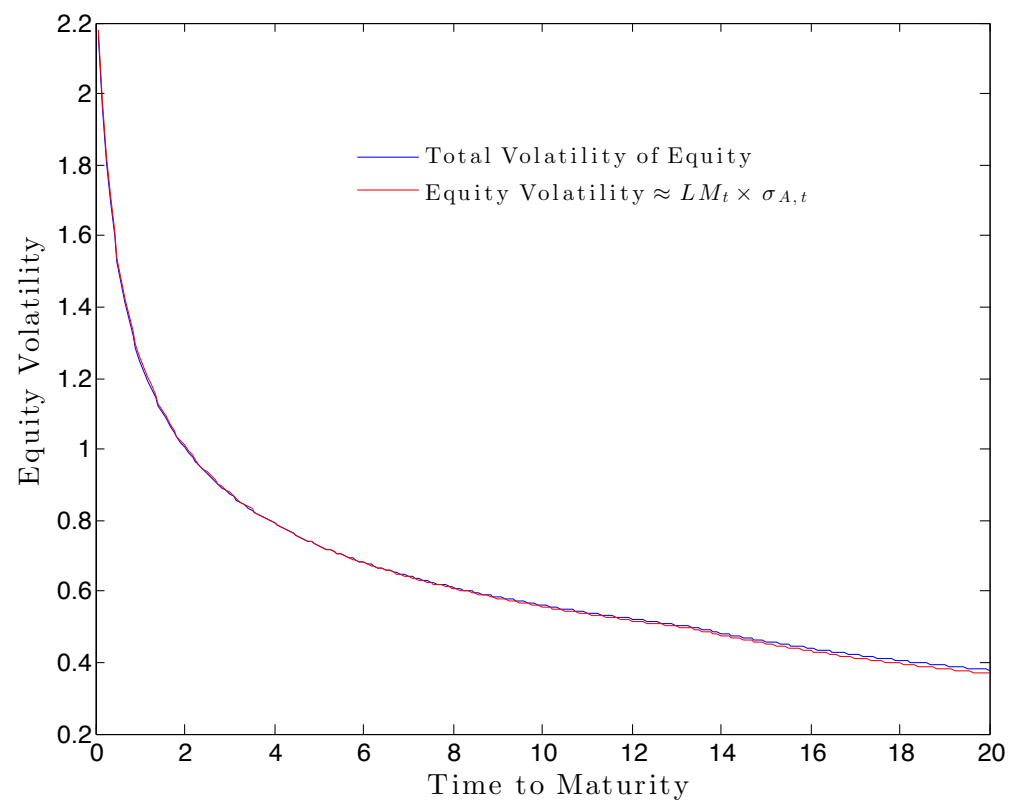

Table 7: Merton (1976) Calibration for Risk-Neutral Asset Prices

\begin{tabular}{|c|c|}
\hline Parameter & Value \\
\hline$\lambda$ & 0.01 \\
\hline$\mu_{J}$ & -0.1 \\
\hline$\sigma_{J}$ & 0.2 \\
\hline
\end{tabular}

Notes: This table provides the parameters we use to calibrate the asset return process under the Merton (1976) model.

In this case, the average jump size $\left(\mu_{J}\right)$ means that when a jump occurs, the asset value falls by $10 \%$, and has a dispersion $\left(\sigma_{J}\right)$ of $20 \%$. Jumps happen at an annualized frequency of $\lambda$, which means there are roughly two expected days per year with jumps for assets returns. In addition, it is well known that the total instantaneous volatility of assets is given by:

$$
\operatorname{vol}_{t}\left(\frac{d A_{t}}{A_{t}}\right)=\sqrt{\sigma_{A}^{2}+\lambda\left[\mu_{J}^{2}+\left(e^{\sigma_{J}^{2}}-1\right)\left(1+\mu_{J}\right)^{2}\right]}
$$

Thus, in order to keep the analysis comparable to the baseline BSM case, we set $\sigma_{A, t}=\sigma_{A}$ such that the total annualized asset volatility is $15 \%$. Using only the leverage multiplier to compute equity volatility (i.e. ignoring volatility added by jumps) is visualized in Figure 16. As is evident from Figure 16, adding jumps to the asset return process has a very small effect on overall equity volatility. The variance of the entire jump process, $J_{A} d N_{A}(t)$, is largely dictated by the variance 
Figure 17: Leverage Multiplier in Merton (1976) Jump-Diffusion Model

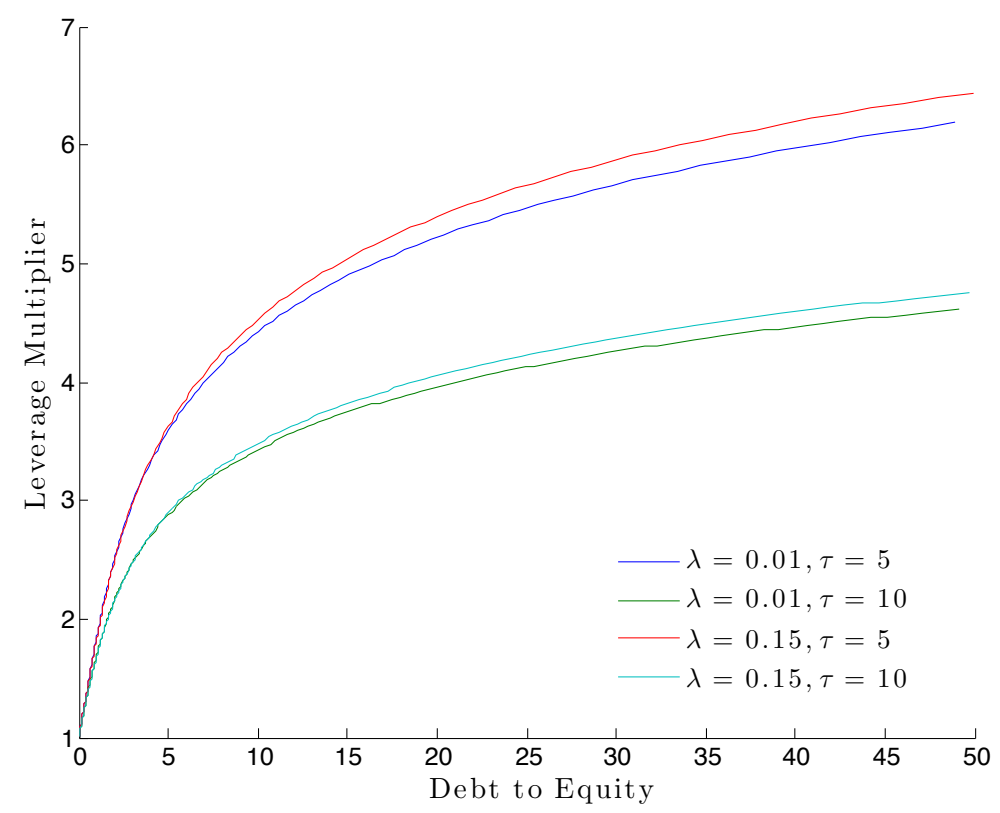

Note: This figure plots the leverage multiplier in the MJD model. Annualized jump intensity takes on one of two values $\lambda \in\{0.01,0.15\}$. The time to maturity of the debt also takes on two possible values $\tau \in\{5,10\}$. The annualized risk-free rate is $r=0.03$.

of the Poisson counting process, which is $\lambda$. Therefore, for reasonable jump arrival intensities, the variance contribution of jumps to equity volatility will be small in magnitude. ${ }^{38}$ Now that we have established that approximating equity volatility by Equation (28), we turn our attention to understanding the properties of the leverage multiplier in this context.

The Leverage Multiplier in the Merton (1976) Model We study the properties of the Merton (1976) leverage multiplier by varying the jump intensity $\lambda$. Again, in order to make these results comparable to the benchmark BSM analysis, we always set $\sigma_{A}$ such that the annualized total volatility of assets is $15 \%$. In addition, we vary the time to maturity of the debt. The leverage multiplier for these different cases is displayed in Figure 17.

The economic underpinnings of the leverage multiplier in the MJD case are, unsurprisingly, quite similar to the BSM case. Holding time to maturity constant, when we decrease the likelihood of a jump, the leverage multiplier also decreases. Since jumps are assumed to decrease the asset value, then a lower likelihood of a jump means the equity is more likely to finish in the money and the effect of leverage on equity volatility is dampened. Holding the likelihood of a jump constant, when we increase the time to maturity, the leverage multiplier increases. In this case, the diffusion

\footnotetext{
${ }^{38}$ In the Online Appendix, we repeat this analysis for other parameterizations and find the conclusions to be robust.
} 
portion of assets dominates the negative jump component (due to the parameter values we chose). Adding maturity to the debt therefore means the equity is more likely to expire with value and so leverage means less for equity volatility. It is likely that for large jump intensities (or large jump sizes) this effect would reverse, but this type of parameterization seems empirically implausible.

\section{A.2.3. Analysis of the Leverage Multiplier with Stochastic Volatility and Jumps}

By now it should be clear that the leverage multiplier takes roughly the same form across different option pricing models; however, for completeness, we conduct one last exploration of the leverage multiplier shape when assets have both stochastic volatility and are subject to jumps (henceforth SVJ). The risk-neutral asset return dynamics are thus described as follows:

$$
\begin{aligned}
\frac{d A_{t}}{A_{t}} & =\left[r-\lambda \mu_{J}\right] d t+\sigma_{A, t} d B_{A}(t)+J d N_{A}(t) \\
d \sigma_{A, t}^{2} & =\kappa\left[\theta-\sigma_{A, t}^{2}\right] d t+\eta \sigma_{A, t} d B_{v}(t)
\end{aligned}
$$

where the jump process for assets retains its original properties as in Equation (20). The closed form solution for option prices under these dynamics is also well-known at this point (e.g. Bakshi, et al. (1997)). We calibrate the model by combining previous parameterizations and repeat them in Table 8.

Table 8: Parameters for Stochastic Volatility with Jumps Model

\begin{tabular}{|c|c|}
\hline Parameter & Value \\
\hline$\kappa$ & 4 \\
\hline$\eta$ & 0.15 \\
\hline$\sigma_{A, t}$ & 0.15 \\
\hline$\rho$ & -0.5 \\
\hline$\lambda$ & 0.01 \\
\hline$\mu_{J}$ & -0.1 \\
\hline$\sigma_{J}$ & 0.2 \\
\hline
\end{tabular}

Notes: This table provides the parameters we use to calibrate the risk-neutral asset return process under the SVJ model used by, among others, Bates (1996) and Bakshi et al. (1997).

The long run average for volatility, $\theta$, set such that the total long run average asset volatility is $15 \%$. As usual, we begin by checking whether the approximation in Equation (28) holds in this setting.

Validating Assumption 1 Figure 18 plots the total equity volatility in the SVJ model against the approximate equity volatility given by (28) for differing maturities and constant leverage. 
Figure 18: Equity Volatility Analysis in SVJ Model

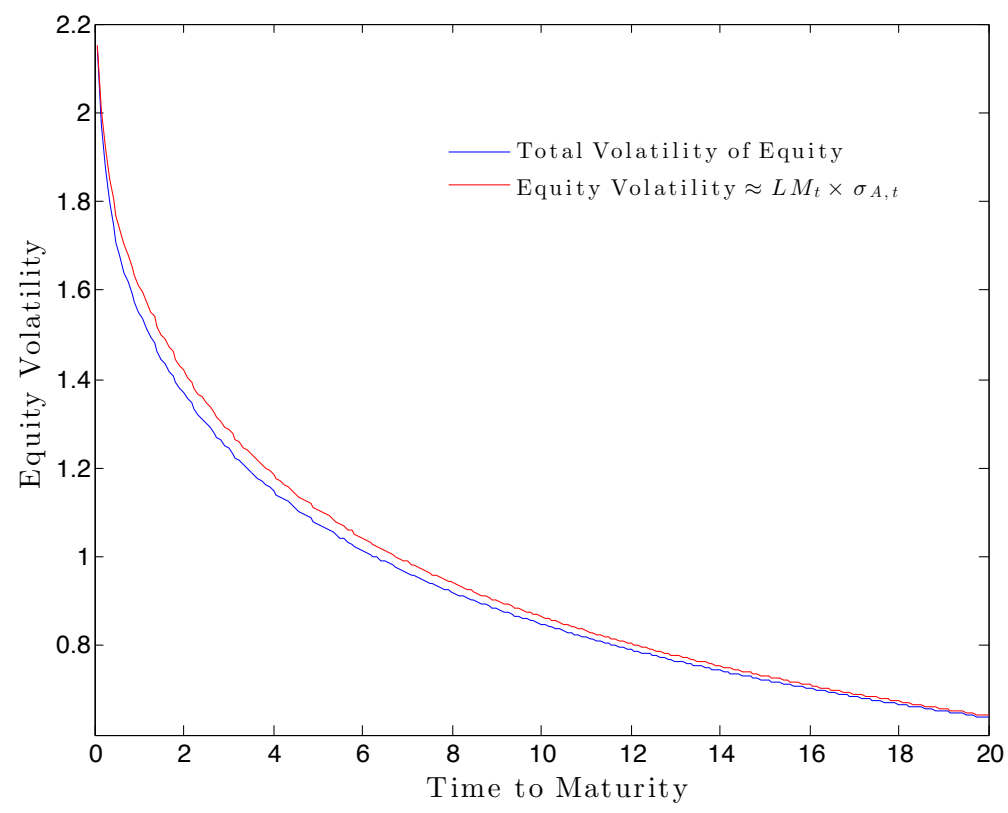

Notes: The blue line in this figure plots the total (true) volatility of equity if assets follow the SVJ model (e.g. Bakshi et al. (1997)). The red line in this figure plots the volatility of equity if it is approximated according to Equation (28). For each time to maturity, we set $A_{t}=1$ and choose the level of debt such that financial leverage is approximately 15. For this analysis, the risk-free rate is set to 0.03 .

At this point, it should not be so surprising that our approximation holds reasonably well. Simply put, for reasonable jump arrival intensities and quickly mean-reverting volatility processes, the main component of equity volatility is asset volatility itself (amplified by the leverage multiplier).

The Leverage Multiplier in the SVJ Model Similarly, Figure 19 plots the leverage multiplier in the SVJ model. As expected, the leverage multiplier in the SVJ cases is the highest for high levels of leverage. The intuition from the BSM model still applies: high leverage means the equity is very likely to expire out of the money for a couple of reasons. First, because volatility and asset return shocks are negatively correlated, the distribution of future asset returns has negative skewness, thus making high levels of leverage even more paralyzing in terms of equity finishing with value. The second reason is due to jumps, which we assume to be negative on average. Together, both factors contribute to a higher equity volatility amplification mechanism when leverage becomes too high. Still, the general shape of the leverage multiplier seems consistent across all of the option pricing models, and we therefore confirm that the results from the main text remain valid. 
Figure 19: Leverage Multiplier in SVJ Model

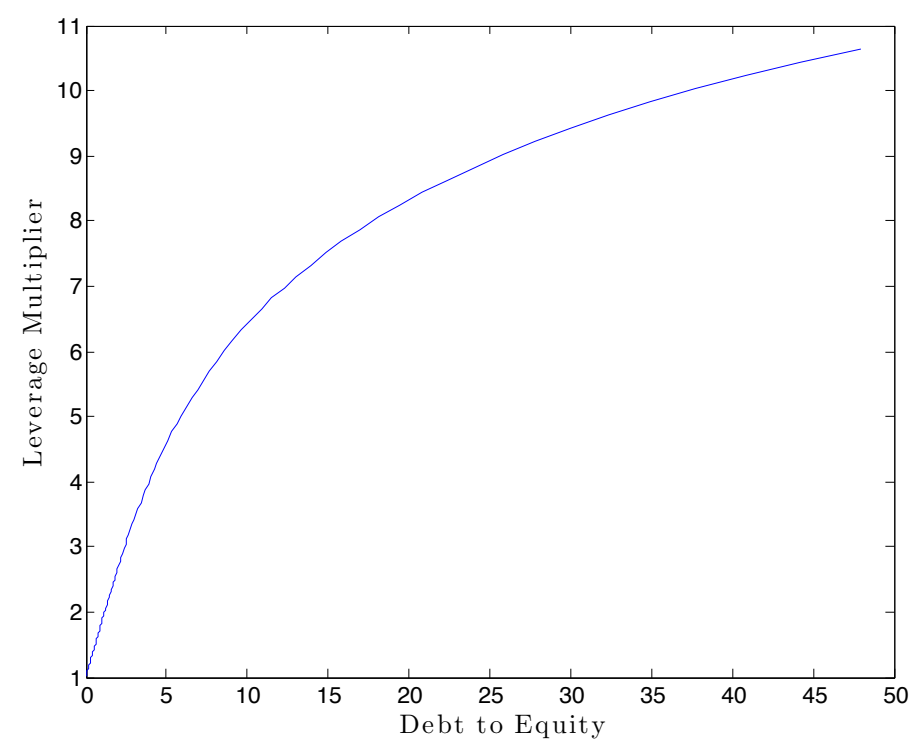

Notes: We plot the leverage multiplier in the SVJ model against leverage. The time to maturity is set to five years and the remaining model parameters are given in Table 8. For this analysis, the risk-free rate is set to $r=0.03$.

\section{B. Appendix: Total Volatility of Equity in Stochastic Volatility with Jump Environment}

We need to compute the variance of the following term:

$$
\begin{aligned}
\mathcal{V}_{A}^{J}\left(J_{A}, N_{A}(t) ; E_{t}\right) & \equiv \operatorname{var}_{t}\left(\left[\frac{E_{t}^{J}-E_{t}}{E_{t}}\right] d N_{A}(t)\right) \\
& =\operatorname{var}_{t}\left(\frac{E_{t}^{J}}{E_{t}} d N_{A}(t)\right)+\operatorname{var}_{t}\left(d N_{A}(t)\right)
\end{aligned}
$$

where we use the independence of $N_{A}(t)$ and $J_{A}$. To make the dependence of equity value on the asset jump more explicit, we replace $E_{t}^{J}$ with the call option pricing function, but we suppress all but the first argument for notional convenience:

$$
E_{t}^{J}=f\left(A_{t}+J_{A}\right)
$$


Using the definition of variance and the properties of the Poisson counting process (i.e. variance is $\lambda)$, we get the following expression for $\mathcal{V}_{A}^{J}\left(J_{A}, N_{A}(t) ; E_{t}\right)$ :

$$
\begin{aligned}
\mathcal{V}_{A}^{J}\left(J_{A}, N_{A}(t) ; A_{t}\right) & =\frac{1}{A_{t}^{2}}\left\{\mathbb{E}\left[f\left(A_{t}+J_{A}\right)^{2} d N(t)^{2}\right]-\mathbb{E}\left[f\left(A_{t}+J_{A}\right) d N(t)\right]^{2}\right\}+\lambda \\
& =\frac{1}{A_{t}^{2}}\left\{\mathbb{E}\left[f\left(A_{t}+J_{A}\right)^{2}\right] \mathbb{E}\left[d N(t)^{2}\right]-\mathbb{E}\left[f\left(A_{t}+J_{A}\right)\right]^{2} \mathbb{E}[d N(t)]^{2}\right\}+\lambda \\
& =\frac{1}{A_{t}^{2}}\left\{\mathbb{E}\left[f\left(A_{t}+J_{A}\right)^{2}\right]\left(\lambda+\lambda^{2}\right)-\mathbb{E}\left[f\left(A_{t}+J_{A}\right)\right]^{2} \lambda^{2}\right\}+\lambda \\
& =\frac{1}{A_{t}^{2}}\left\{\psi\left(A_{t}, J_{A}\right)\left(\lambda+\lambda^{2}\right)-\Gamma\left(A_{t}, J_{A}\right) \lambda^{2}\right\}+\lambda
\end{aligned}
$$

Here, the second line uses the independence of $N_{A}(t)$ and $J_{A}$, and the third line uses the standard variance definition for a Poission process. ${ }^{39} \psi(\cdot)$ and $\Gamma(\cdot)$ are given by:

$$
\begin{aligned}
\psi\left(A_{t}, J_{A}\right) & =\mathbb{E}\left[f\left(A_{t}+J_{A}\right)^{2}\right] \\
& =\int_{-\infty}^{\infty} f\left(A_{t}+e^{y}-1\right)^{2} h_{Y}(y) d y \\
\Gamma\left(E_{t}, J_{A}\right) & =\left[\int_{-\infty}^{\infty} f\left(A_{t}+e^{y}-1\right) h_{Y}(y) d y\right]^{2}
\end{aligned}
$$

where $h_{Y}(y)$ is the pdf of a normal random variable with mean $\log \left[1+\mu_{J}\right]-\sigma_{J}^{2} / 2$ and variance $\sigma_{J}^{2}$. In practice, $\mathcal{V}_{A}^{J}\left(J_{A}, N_{A}(t) ; A_{t}\right)$ is easily computed numerically.

\footnotetext{
39 i.e.

$$
\begin{aligned}
\operatorname{var}_{t}\left(d N_{A}(t)\right) & =\mathbb{E}\left[d N_{A}(t)^{2}\right]-\mathbb{E}\left[d N_{A}(t)\right]^{2} \\
& \Leftrightarrow \\
\mathbb{E}\left[d N_{A}(t)^{2}\right] & =\operatorname{var}_{t}\left(d N_{A}(t)\right)+\mathbb{E}\left[d N_{A}(t)\right]^{2} \\
& =\lambda+\lambda^{2}
\end{aligned}
$$
}




\section{Appendix: Empirical Argument for Ignoring Volatil- ity Terms}

For exposition, we repeat Equation (3):

$$
\frac{d E_{t}}{E_{t}}=\Delta_{t} \cdot \frac{A_{t}}{D_{t}} \cdot \frac{D_{t}}{E_{t}} \cdot \frac{d A_{t}}{A_{t}}+\frac{\partial f}{\partial \sigma_{A, t}^{f}} \cdot \frac{D_{t}}{E_{t}} \cdot d \sigma_{A, t}^{f}
$$

It is straightforward to work out that equity variance will be as follows:

$$
\begin{aligned}
\operatorname{var}_{t}\left(\frac{d E_{t}}{E_{t}}\right)= & \left(\Delta_{t} \frac{A_{t}}{D t} \cdot \frac{D_{t}}{E_{t}}\right)^{2} \operatorname{var}_{t}\left(\frac{d A_{t}}{A_{t}}\right)+\left(\frac{\nu_{t} D_{t}}{E_{t}}\right)^{2} \operatorname{var}_{t}\left(d \sigma_{A, t}^{f}\right) \\
& +2\left(\Delta_{t} \frac{A_{t}}{D t} \cdot \frac{D_{t}}{E_{t}}\right)\left(\frac{\nu_{t} D_{t}}{E_{t}}\right) \sqrt{\operatorname{var}_{t}\left(\frac{d A_{t}}{A_{t}}\right) \operatorname{var}_{t}\left(d \sigma_{A, t}^{f}\right)} \times \rho_{t}\left(\frac{d A_{t}}{A_{t}}, d \sigma_{A, t}^{f}\right)
\end{aligned}
$$

where $\Delta_{t}=\partial f\left(A_{t} / D_{t}, 1, \sigma_{A, t}^{f}, \tau, r\right) / \partial A_{t}$ and $\nu_{t}=\partial f\left(A_{t} / D_{t}, 1, \sigma_{A, t}^{f}, \tau, r\right) / \partial d \sigma_{A, t}^{f}$. In option pricing lingo, $\Delta_{t}$ is the "delta" of the call option on assets, and $\nu_{t}$ is the "vega" of the call option on assets. ${ }^{40}$ In the model where we set the long run volatility of assets to be the unconditional volatility of the asset GJR process, this analysis is moot as $d \sigma_{A, t}^{f}=0$. Our task now is to show that the last two terms are negligible for the purposes of modeling equity volatility, when we use the GJR forecast for long run asset volatility.

\section{C.1. Magnitude of Volatility Terms}

In the language of the Structural GARCH model we can simply substitute $L M_{t}$ into Equation (31) where it is appropriate:

$$
\begin{aligned}
\operatorname{var}_{t}\left(\frac{d E_{t}}{E_{t}}\right)= & \left(L M_{t}\right)^{2} \operatorname{var}_{t}\left(\frac{d A_{t}}{A_{t}}\right)+\left(\frac{\nu_{t} D_{t}}{E_{t}}\right)^{2} \operatorname{var}_{t}\left(d \sigma_{A, t}^{f}\right) \\
& +2\left(L M_{t}\right)\left(\frac{\nu_{t} D_{t}}{E_{t}}\right) \sqrt{\operatorname{var}_{t}\left(\frac{d A_{t}}{A_{t}}\right) \operatorname{var}_{t}\left(d \sigma_{A, t}^{f}\right)} \times \rho_{t}\left(\frac{d A_{t}}{A_{t}}, d \sigma_{A, t}^{f}\right)
\end{aligned}
$$

In order to investigate the magnitude of the terms we ignore (i.e. any term containing $\nu_{t}$ ), we need a functional form for the sensitivity of the equity value to changes in long run asset volatility. Since we are only interested in magnitudes, we will use the Black-Scholes vega. It is unlikely that the Black-Scholes vega is incorrect by an order of magnitude, so for this exercise it will be sufficient. The next thing we need in order to quantitatively evaluate Equation (32) are time-series for $L M_{t}$, $d A_{t} / A_{t}$, and $d \sigma_{A, t}^{f}$. To be precise, if we extended the model to include changes in volatility we would

\footnotetext{
${ }^{40}$ To be precise, these are the delta and vega of the option where debt has been normalized to 1.
} 
undoubtedly obtain different estimates for these three quantities. Again, since our goal is to assess relative magnitudes, we will simply use the values delivered by our Structural GARCH model for $L M_{t}, d A_{t} / A_{t}$, and $d \sigma_{A, t}^{f}$. Formally, we define $d \sigma_{A, t}^{f}$ as:

$$
d \sigma_{A, t}^{f}=\sqrt{h_{A, t+1}^{f} / \tau}-\sqrt{h_{A, t}^{f} / \tau}
$$

where $h_{A, t+1}^{f}$ is the forecast of total volatility over the life of the option. Finally, we set the volatility of volatility to be constant and the correlation between asset returns and volatility to be constant. Under this assumption, we estimate these quantities using their in-sample moments.

\section{C.1.1. Case Study: JPM}

As an example, we study JPM. Table 9 contains the in-sample moments of the estimated stochastic volatility process.

Table 9: Moments for Volatility Forecast Innovations

\begin{tabular}{|c|c|}
\hline Variable & Value \\
\hline$\sqrt{\operatorname{var}_{t}\left(d \sigma_{A, t}^{f}\right)}$ & $4.9737 \mathrm{e}-04$ \\
\hline$\rho_{t}\left(\frac{d A_{t}}{A_{t}}, d \sigma_{A, t}^{f}\right)$ & -0.427 \\
\hline
\end{tabular}

Notes: This table contains the in-sample moments of the asset volatility and asset return process implied by the Structural GARCH applied to JPM.

Unsurprisingly, there is a strong negative correlation between the innovation to the long run asset volatility forecast and asset returns. In addition, the extremely small volatility of volatility provides us our first piece of supporting evidence in favor of ignoring the additional vega terms. Next, we plot each of the three terms from Equation (32). Figure 20 confirms our assumption that including $d \sigma_{A, t}^{f}$ has a small effect on our main volatility specification. Moreover, it is clear from Equation (32) that any additional volatility of volatility that contributes to equity volatility will be offset by the negative correlation between asset volatility and asset returns. On average, the variance arising from our standard leverage multiplier term is approximately 12 times larger (in absolute value) than the sum the additional terms due to vega. Furthermore, the leverage multiplier term dominates the vega terms in times of high volatility, which tend to be our main areas of interest. Thus, we conclude that we can ignore the additional vega terms within the context of our Structural GARCH model. 
Figure 20: Decomposition of Equity Variance for JPM

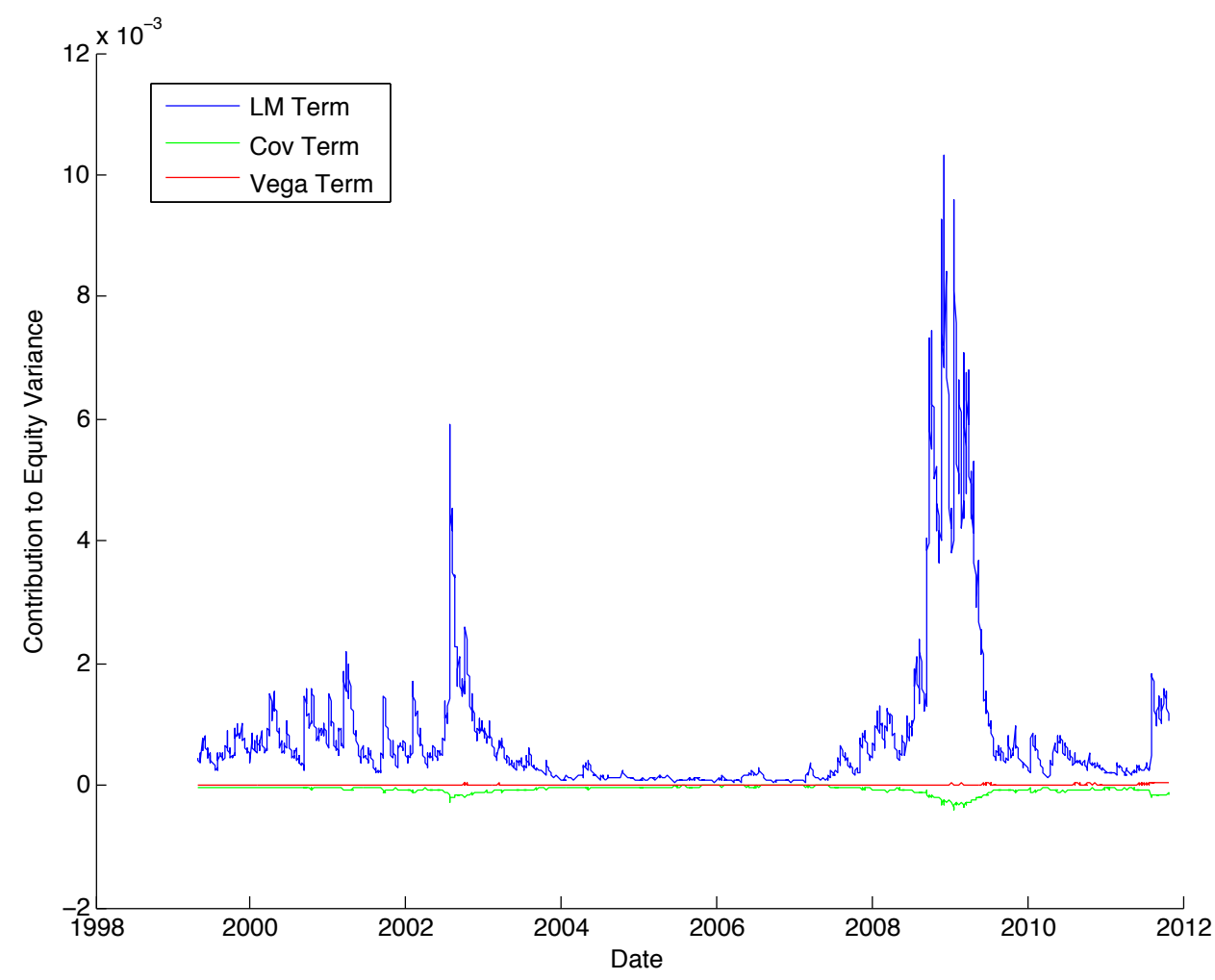

Notes: The figure above plots each time series for each of the three terms in Equation (32). The blue line represents the first term, containing $L M_{t}^{2}$. The red line is the second term, and contains only $\operatorname{var}_{t}\left(d \sigma_{A, t}^{f}\right)$. Finally, the green line is last covariance term. 


\section{Data Appendix}

\section{D.1. List of Firms Analyzed}

Table 10: List of Firms Analyzed

\begin{tabular}{|l|l|l|l|}
\hline ABK & CBSS & HRB & SAF \\
ACAS & CFC & HUM & SEIC \\
AET & CI & JNS & SLM \\
AFL & CIT & JPM & SNV \\
AGE & CMA & KEY & SOV \\
AIG & CNA & LEH & STI \\
ALL & COF & LNC & STT \\
AMP & CVH & MBI & TMK \\
AMTD & ETFC & MER & TROW \\
AON & ETN & MET & TRV \\
AXP & EV & MI & UB \\
BAC & FITB & MMC & UNM \\
BBT & FNF & MS & USB \\
BEN & FNM & MTB & WB \\
BK & FRE & NCC & WFC \\
BLK & GNW & PBCT & WM \\
BOT & GS & PFG & WRB \\
BSC & HBAN & PGR & ZION \\
C & HCBK & PNC & \\
CB & HIG & PRU & \\
CBH & HNT & RF & \\
\hline
\end{tabular}

\section{D.2. Outlier Cases}

Compared to the estimation done on $11 / 11 / 2013$, there were a few firms that had name changes. To avoid issues with data quality, we throw these out. This leaves us with a total of 82 firms. There are 10 firms that hit the lower bound: SCHW:US, JNS:US, LM:US, BK:US, BLK:US, NTRS:US, CME:US, CINF:US, TMK:US, UNH:US. 